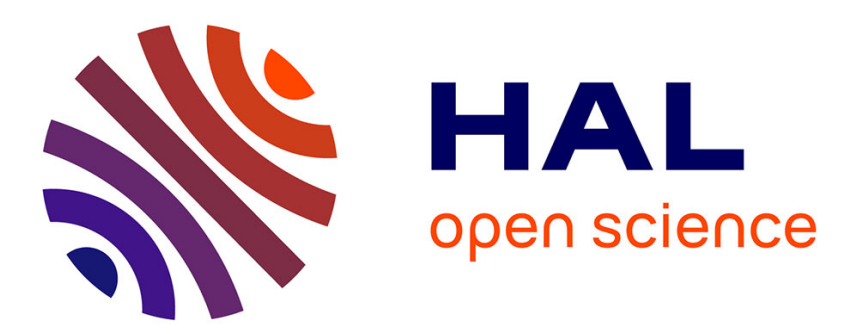

\title{
Modeling and Analysis of the Coupling in Discrete Fracture Matrix models
}

Martin J. Gander, Julian Hennicker, Roland Masson

\section{To cite this version:}

Martin J. Gander, Julian Hennicker, Roland Masson. Modeling and Analysis of the Coupling in Discrete Fracture Matrix models. SIAM Journal on Numerical Analysis, 2021, 59 (1), pp.195-218. 10.1137/20M1312125. hal-02437030

\section{HAL Id: hal-02437030 https://hal.science/hal-02437030}

Submitted on 13 Jan 2020

HAL is a multi-disciplinary open access archive for the deposit and dissemination of scientific research documents, whether they are published or not. The documents may come from teaching and research institutions in France or abroad, or from public or private research centers.
L'archive ouverte pluridisciplinaire HAL, est destinée au dépôt et à la diffusion de documents scientifiques de niveau recherche, publiés ou non, émanant des établissements d'enseignement et de recherche français ou étrangers, des laboratoires publics ou privés. 


\title{
Modeling and Analysis of the Coupling in Discrete Fracture Matrix models
}

\author{
Martin J. Gander*, Julian Hennicker*, Roland Masson ${ }^{\dagger}$ \\ January 13, 2020
}

\begin{abstract}
This paper deals with the derivation and analysis of reduced order elliptic PDE models on fractured domains. We use a Fourier analysis to obtain coupling conditions between subdomains, when the fracture is represented as a hypersurface embedded in the surrounded rock matrix. We compare our results to prominent examples from the literature, for diffusive models. In a second step, we present error estimates for the reduced order models in terms of the fracture width. For the proofs, we rely on a combination of Fourier analysis, asymptotic expansions and functional analysis. Finally, we study the behaviour of the error of the reduced order solutions on numerical test cases, when the fracture width tends to zero.
\end{abstract}

\section{Introduction}

There are countless physical processes in real life applications that can be modeled by elliptic partial differential equations. One of the difficulties one often encounters in the formulation of such models is the presence of heterogeneities in the ambient media (i.e. spatially discontinuous coefficients in the differential equations). If this is the case, then one has to think about coupling conditions between regions at the material interfaces. A further difficulty for the numerical solution of the problem occurs when the heterogeneity is a thin layer, in the sense that its length is much bigger than its width, which requires anisotropic and/or very small mesh cells inside the layer. This situation most prominently occurs in subsurface flow applications, where the thin heterogeneous layers are called fractures, and they are surrounded by the so-called rock matrix. Being motivated by such applications, we adopt this nomenclature in what follows.

A model reduction strategy consists in representing the fractures as hypersurfaces, embedded in the matrix domain. This results in a system of PDEs in the full dimensional matrix domain coupled with a system of tangential PDEs in the reduced dimensional fractures. Therefore, these models are called in the literature mixed- or hybrid-dimensional models, or Discrete Fracture Matrix (DFM) models. A common method to establish such models consists in integrating the fracture equations over the fracture width and using some ad hoc approximations for the coupling conditions (see $[9,19,1,18]$, where these techniques have been employed on simple geometries and $[4,6]$ for extensions to general fracture networks). For non linear DFM models, we refer to $[5,2,7]$. For an overview and comparison of current discretisation methods, see [3].

The convergence of the DFM model solution to the full model solution has been the objective of several studies. They all carry out the convergence proofs in a classical functional analysis setting, but they differ in the asymptotic scaling of the model parameters, which leads to different DFM models. In [22], the authors use a suitable modification of the solution inside

*Université de Genève, 2-4 rue du Lièvre, CP 64, CH-1211 Genève, \{martin.gander, julian.hennicker\}@unige.ch

†Laboratoire J.A. Dieudonné, UMR 7351 CNRS, Université Nice Sophia Antipolis, et quipe COFFEE, INRIA Sophia Antipolis Méditerranée, Parc Valrose 06108 Nice Cedex 02, France, roland.masson@unice.fr 
the fracture, in order to control its gradient and to show convergence for isotropic Darcy flow and a fracture of high resistivity. In [8], the authors also use a modification of the fracture solution, and derive error estimates for anisotropic Darcy flow. In [20], a rescaling of the fracture normal coordinate is used, which yields a rescaled model on a geometry independent of the fracture width. The convergence is then proved by using the compactness of the rescaled solution. This technique is further applied in $[21,17]$ to non linear problems (Richards equation and reactive transport). On the other hand, in [7] it has been observed numerically that the well established approximation of pressure continuity accross highly conductive fractures for the linear single phase flow models leads to non convergent solutions for two phase flow models, which involve highly non linear matrix fracture transmission conditions. Analytical results for these models are still missing.

In the present paper, we use a completely new approach to develop and analyze reduced order models for general linear elliptic problems. Our focus lies on the derivation of coupling conditions, which have to be satisfied by the traces of the solutions for the matrix domain on each side of the matrix-fracture interfaces. We emphasize that we are not only interested in the derivation of coupling conditions that have to be satisfied in the limit of vanishing aperture, but in particular with the derivation of coupling conditions that have to hold up to a certain order of the aperture, which in turn occurs as a model parameter. The idea is to first use a Fourier transform in the fracture tangential direction, which allows us to eliminate the fracture unknowns and to derive exact coupling conditions between the matrix subdomains, a technique which can be regarded as a continuous analogue to a Schur complement of the fracture unknowns onto the matrix-fracture interfaces, and which is frequently used in domain decomposition to derive coupling conditions for optimal or optimized Schwarz methods, see [10, 11] and references therein, and $[12,13,14,15]$ for a different type of heterogeneous coupling using such techniques. Reduced order coupling conditions are then obtained by truncating the asymptotic expansions of the exact conditions at the desired order.

Our paper is organized as follows: in Section 2, we present the model problem for which we develop coupling conditions. Section 3 is devoted to the derivation of the reduced order models, and Section 4 to the study of their well-posedness. The focus of Section 5 is a posteriori approximations of the fracture unknowns by suitable interpolations of the traces of the matrix solution at the interfaces. A comparison of our new reduced models to the Darcy flow models proposed in [19] is given in Section 6, where we also recover and extend the convergence results from [22]. In Section 7, we derive error estimates for reduced order models for anisotropic diffusion problems, which are sharper than the estimates given in [8]. From the exact and reduced order coupling conditions in Fourier space, we can infer the error of the traces of the matrix Fourier coefficients at the interfaces. Using trace and expansion inequalities for functions in fractional Sobolev spaces, we obtain error estimates of optimal order in the fracture width, namely cubic order of convergence for the $H^{1}$-norm of the matrix solutions and quadratic order of convergence for the $H^{\frac{1}{2}}$-norm of the fracture solution. In Section 8, we present a series of numerical tests, where we address the convergence of the reduced order solutions w.r.t. the fracture width, for different asymptotic behavior of the model parameters, including cases not covered by our analysis. 


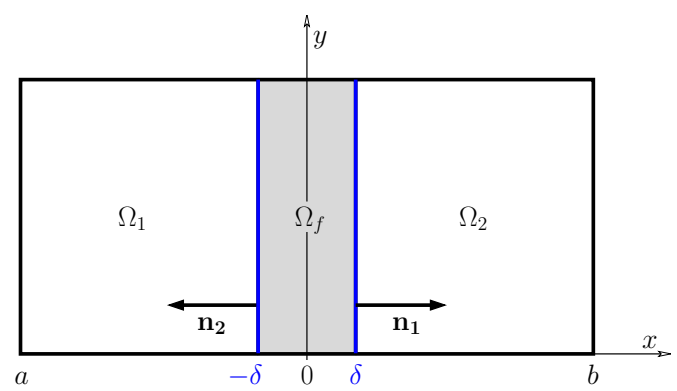

Figure 1: Model problem domain, where $\Omega_{1}=\left(a_{x},-\delta\right) \times \Gamma, \Omega_{2}=\left(\delta, b_{x}\right) \times \Gamma$ and $\Omega_{f}=(-\delta, \delta) \times \Gamma$, with $a_{x}, b_{x} \in \mathbb{R}$ and $\Gamma=\left(a_{y}, b_{y}\right) \subset \mathbb{R}$. Note that the Fourier analysis in sections 3 and 7 , will be carried out on unbounded domains by setting $\Gamma=\mathbb{R}$. The unit normals on $\Gamma$ pointing outside of $\Omega_{j}$ are denoted by $\mathbf{n}_{j}$.

\section{Model problem}

We consider as our model problem a fracture between two matrix domains as illustrated in Figure 1,

$$
\begin{aligned}
& -\operatorname{divq}_{j}+\frac{\mathbf{b}_{j}}{2} \cdot \nabla u_{j}+\left(\eta_{j}-\operatorname{div} \frac{\mathbf{b}_{j}}{2}\right) u_{j}=h_{j} \quad \text { in } \quad \Omega_{j}, j=1,2, f, \\
& \mathbf{q}_{j}=\left(\mathbf{A}_{j} \nabla-\frac{\mathbf{b}_{j}}{2}\right) u_{j} \quad \text { in } \quad \Omega_{j}, j=1,2, f, \\
& u_{j}=u_{f} \quad \text { on } \quad \partial \Omega_{j} \cap \partial \Omega_{f}, j=1,2, \\
& \mathbf{q}_{j} \cdot \mathbf{n}_{j}=\mathbf{q}_{f} \cdot \mathbf{n}_{j} \quad \text { on } \quad \partial \Omega_{j} \cap \partial \Omega_{f}, j=1,2,
\end{aligned}
$$

together with some suitable outer boundary conditions. The model coefficients are $\eta_{j}: \Omega_{j} \rightarrow$ $\mathbb{R}_{\geq 0}, \mathbf{b}_{j}: \Omega_{j} \rightarrow \mathbb{R}^{2}$, such that $\eta_{j}-\operatorname{divb}_{j} \geq 0$, and coercive matrices $\mathbf{A}_{j}: \Omega_{j} \rightarrow \mathbb{R}^{2 \times 2}$. The model unknowns are $\mathbf{q}_{j}$ and $u_{j}$. For simplicity, we assume that the fracture source term is trivial, $h_{f} \equiv 0$.

\section{Derivation of the reduced models by Fourier analysis}

We now derive suitable coupling conditions between the matrix domains $\Omega_{1}$ and $\Omega_{2}$ that complement the matrix equations

$$
\begin{array}{rlrl}
-\operatorname{divq}_{j}+\frac{\mathbf{b}_{j}}{2} \cdot \nabla u_{j}+\left(\eta_{j}-\operatorname{div} \frac{\mathbf{b}_{j}}{2}\right) u_{j} & =h_{j} & \text { in } \quad \Omega_{j}, j=1,2, \\
\mathbf{q}_{j} & =\left(\mathbf{A}_{j} \nabla-\frac{\mathbf{b}_{j}}{2}\right) u_{j} & & \text { in } \quad \Omega_{j}, j=1,2,
\end{array}
$$

and allow us to find approximate matrix solutions $u_{j}^{\text {red }}, \mathbf{q}_{j}^{\text {red }}, j=1,2$, for small fracture apertures $\delta>0$, without solving the fracture equations. The fracture solution can then be reconstructed a posteriori from the traces at the interfaces of the matrix solutions, as discussed in Section 5.

In what follows, we will drop the index $f$ for fracture parameters whenever it does not lead to confusion. Only in Section 4, we will have to use it again.

We assume now for simplicity that the overall domain is $\mathbb{R}^{2}$ to be able to use Fourier transforms (similar results could also be obtained on bounded domains using Fourier series). From (1) and (2) with $h_{f} \equiv 0$, the Fourier coefficients $\hat{u}_{f}(x, k)$ of $u_{f}(x, y)$ have to satisfy for all $k \in \mathbb{R}$ the ordinary differential equation

$$
-a_{11} \partial_{x x} \hat{u}_{f}+\left(b_{1}-\left(a_{12}+a_{21}\right) i k\right) \partial_{x} \hat{u}_{f}+\left(a_{22} k^{2}+b_{2} i k+\eta\right) \hat{u}_{f}=0 \quad \text { in } \quad \Omega_{f} .
$$


The roots of the characteristic polynomial associated with (7) are $\lambda_{1,2}=r \pm s$, where

$$
r=-\frac{1}{2 a_{11}}\left(\left(a_{12}+a_{21}\right) i k-b_{1}\right) \quad \text { and } \quad s=\left(r^{2}+\frac{1}{a_{11}}\left(a_{22} k^{2}+b_{2} i k+\eta\right)\right)^{\frac{1}{2}} .
$$

The ansatz for the solution of (7),

$$
\hat{u}_{f}(x, k)=A(k) e^{\lambda_{1} x}+B(k) e^{\lambda_{2} x},
$$

together with the coupling conditions (3) and (4) yields for the Fourier coefficients $\hat{u}_{j}(x, k)$ of $u_{j}(x, y)$ and $\hat{\mathbf{q}}_{j}(x, k)$ of $\mathbf{q}_{j}(x, y), j=1,2$, on the interfaces,

$$
\begin{aligned}
\hat{u}_{1}(-\delta, k) & =A(k) e^{-\delta \lambda_{1}}+B(k) e^{-\delta \lambda_{2}}, \\
\hat{u}_{2}(\delta, k) & =A(k) e^{\delta \lambda_{1}}+B(k) e^{\delta \lambda_{2}}, \\
\hat{\mathbf{q}}_{1}(-\delta, k) \cdot \mathbf{n}_{1} & =a_{11} \lambda_{1} A(k) e^{-\delta \lambda_{1}}+a_{11} \lambda_{2} B(k) e^{-\delta \lambda_{2}}+\left(a_{12} i k-\frac{b_{1}}{2}\right) \hat{u}_{1}(-\delta, k), \\
-\hat{\mathbf{q}}_{2}(\delta, k) \cdot \mathbf{n}_{2} & =a_{11} \lambda_{1} A(k) e^{\delta \lambda_{1}}+a_{11} \lambda_{2} B(k) e^{\delta \lambda_{2}}+\left(a_{12} i k-\frac{b_{1}}{2}\right) \hat{u}_{2}(\delta, k) .
\end{aligned}
$$

Equations (9) and (10) are now solved for $A$ and $B$,

$$
A(k)=\frac{\hat{u}_{2}(\delta, k) e^{-\delta \lambda_{2}}-\hat{u}_{1}(-\delta, k) e^{\delta \lambda_{2}}}{2 \sinh (2 s \delta)}, \quad B(k)=\frac{\hat{u}_{1}(-\delta, k) e^{\delta \lambda_{1}}-\hat{u}_{2}(\delta, k) e^{-\delta \lambda_{1}}}{2 \sinh (2 s \delta)},
$$

which can then be substituted into the remaining two equations (11) and (12). After some calculations, this leads to the exact coupling conditions between $\hat{u}_{1}, \hat{\mathbf{q}}_{1}$ in $\Omega_{1}$ and $\hat{u}_{2}, \hat{\mathbf{q}}_{2}$ in $\Omega_{2}$ across the fracture which has been eliminated,

$$
\begin{aligned}
\sinh (2 s \delta) \hat{\mathbf{q}}_{1}(-\delta) \cdot \mathbf{n}_{1}+ & \left(a_{11} s \cosh (2 s \delta)+\rho \sinh (2 s \delta)\right) \hat{u}_{1}(-\delta) \\
& =a_{11} s e^{-2 \delta r} \hat{u}_{2}(\delta) \\
\sinh (2 s \delta) \hat{\mathbf{q}}_{2}(\delta) \cdot \mathbf{n}_{2}+ & \left(a_{11} s \cosh (2 s \delta)-\rho \sinh (2 s \delta)\right) \hat{u}_{2}(\delta) \\
& =a_{11} s e^{2 \delta r} \hat{u}_{1}(-\delta)
\end{aligned}
$$

where $\rho=\frac{a_{21}-a_{12}}{2} i k$. Note that even without the fracture, (14) and (15) together with (5) and (6) form a model with identical matrix solutions to the original model (1)-(4). For the remaining part of this section, we will drop the arguments indicating the evaluation at $x=-\delta$ for the functions living in $\Omega_{1}$ and at $x=\delta$ for those living in $\Omega_{2}$. Taking the sum $(14)+(15)$ yields an expression related to the normal velocity jump across the fracture, whereas the difference (14) - (15) gives an expression related to the pressure jump across the fracture,

$$
\begin{aligned}
& -\sinh (2 s \delta)\left(\hat{\mathbf{q}}_{2} \cdot \mathbf{n}_{2}+\hat{\mathbf{q}}_{1} \cdot \mathbf{n}_{1}\right) \\
& \quad=a_{11} s\left(\cosh (2 s \delta)\left(\hat{u}_{1}+\hat{u}_{2}\right)-\left(e^{2 \delta r} \hat{u}_{1}+e^{-2 \delta r} \hat{u}_{2}\right)\right)+\rho \sinh (2 s \delta)\left(\hat{u}_{1}-\hat{u}_{2}\right), \\
& a_{11} s\left(\cosh (2 s \delta)\left(\hat{u}_{2}-\hat{u}_{1}\right)+\left(e^{-2 \delta r} \hat{u}_{2}-e^{2 \delta r} \hat{u}_{1}\right)\right) \\
& =\sinh (2 s \delta)\left(\hat{\mathbf{q}}_{1} \cdot \mathbf{n}_{1}-\hat{\mathbf{q}}_{2} \cdot \mathbf{n}_{2}\right)+\rho \sinh (2 s \delta)\left(\hat{u}_{1}+\hat{u}_{2}\right) .
\end{aligned}
$$

We now expand (16), (17) into a series in $\delta$ and truncate at a given order. We then obtain the following reduced order coupling conditions at $x= \pm \delta$ :

1. Truncation after the leading-order term ( $\mathrm{CC} 0$ coupling conditions):

$$
\hat{\mathbf{q}}_{2}^{\text {red }} \cdot \mathbf{n}_{2}+\hat{\mathbf{q}}_{1}^{\text {red }} \cdot \mathbf{n}_{1}=0 \quad \text { and } \quad \hat{u}_{2}^{\text {red }}-\hat{u}_{1}^{\text {red }}=0 .
$$

2. Truncation after the next-to-leading-order term (CC1 coupling conditions):

$$
\begin{aligned}
& -\left(\hat{\mathbf{q}}_{2}^{\text {red }} \cdot \mathbf{n}_{2}+\hat{\mathbf{q}}_{1}^{\text {red }} \cdot \mathbf{n}_{1}\right)=\delta\left(a_{22} k^{2}+b_{2} i k+\eta\right)\left(\hat{u}_{1}^{\text {red }}+\hat{u}_{2}^{\text {red }}\right)+\left(-a_{21} i k+\frac{b_{1}}{2}\right)\left(\hat{u}_{2}^{\text {red }}-\hat{u}_{1}^{\text {red }}\right), \\
& \delta\left(\hat{\mathbf{q}}_{1}^{\text {red }} \cdot \mathbf{n}_{1}-\hat{\mathbf{q}}_{2}^{\text {red }} \cdot \mathbf{n}_{1}\right)=a_{11}\left(\hat{u}_{2}^{\text {red }}-\hat{u}_{1}^{\text {red }}\right)+\delta\left(a_{12} i k-\frac{b_{1}}{2}\right)\left(\hat{u}_{1}^{\text {red }}+\hat{u}_{2}^{\text {red }}\right) .
\end{aligned}
$$


To get back to the physical unknowns $u_{j}$ and $\mathbf{q}_{j}, j=1,2$, we perform an inverse Fourier transform by formally applying the rules,

$$
\hat{u}_{j}^{\mathrm{red}} \mapsto u_{j}^{\mathrm{red}}, \quad \hat{\mathbf{q}}_{j}^{\mathrm{red}} \mapsto \mathbf{q}_{j}^{\mathrm{red}}, \quad k^{2} \mapsto-\partial_{y y}, \quad i k \mapsto \partial_{y} .
$$

We therefore obtain as reduced order approximations of the exact coupling conditions between the matrix domains $\Omega_{1}$ and $\Omega_{2}$

1. CC0 coupling conditions:

$$
\mathbf{q}_{1}^{\text {red }} \cdot \mathbf{n}_{1}+\mathbf{q}_{2}^{\text {red }} \cdot \mathbf{n}_{2}=0 \quad \text { and } \quad u_{2}^{\text {red }}-u_{1}^{\text {red }}=0 .
$$

2. CC1 coupling conditions:

$$
\begin{aligned}
& -\left(\mathbf{q}_{1}^{\text {red }} \cdot \mathbf{n}_{1}+\mathbf{q}_{2}^{\text {red }} \cdot \mathbf{n}_{2}\right)=\delta\left(-a_{22} \partial_{y y}+b_{2} \partial_{y}+\eta\right)\left(u_{1}^{\text {red }}+u_{2}^{\text {red }}\right)+\left(-a_{21} \partial_{y}+\frac{b_{1}}{2}\right)\left(u_{2}^{\text {red }}-u_{1}^{\text {red }}\right) \\
& \delta\left(\mathbf{q}_{1}^{\text {red }} \cdot \mathbf{n}_{1}-\mathbf{q}_{2}^{\text {red }} \cdot \mathbf{n}_{2}\right)=a_{11}\left(u_{2}^{\text {red }}-u_{1}^{\text {red }}\right)+\delta\left(a_{12} \partial_{y}-\frac{b_{1}}{2}\right)\left(u_{1}^{\text {red }}+u_{2}^{\text {red }}\right) .
\end{aligned}
$$

Remark 3.1 Since the CCO coupling conditions represent the trivial case without any fracture, we will not consider this model further. We could also derive higher order coupling conditions by using higher order expansions, but such models would in general not be well posed.

\section{Well-posedness of the reduced models}

In this section, we show the well-posedness of the reduced models on bounded domains. First, we have to introduce the trace operators

$$
\gamma_{j}: H^{1}\left(\Omega_{j}\right) \rightarrow L^{2}(\Gamma) \quad \text { and } \quad \gamma_{j, \partial \Omega}: H^{1}\left(\Omega_{j}\right) \rightarrow L^{2}\left(\partial \Omega_{j} \backslash(\{ \pm \delta\} \times \Gamma)\right)
$$

and the normal trace operators

$$
\gamma_{\mathbf{n}_{j}}: H_{\operatorname{div}}\left(\Omega_{j}\right) \rightarrow H^{-\frac{1}{2}}(\Gamma)
$$

for $j=1,2$, with $\mathbf{n}_{1}=\mathbf{n}$ and $\mathbf{n}_{2}=-\mathbf{n}$. and the function spaces

$$
\begin{aligned}
V_{j} & :=\left\{\varphi_{j} \in H^{1}\left(\Omega_{j}\right) \mid \gamma_{j, \partial \Omega} \varphi_{j}=0\right\} & \text { for } j=1,2, \\
V & :=\left\{\left(\varphi_{1}, \varphi_{2}\right) \in V_{1} \oplus V_{2} \mid \gamma_{1} \varphi_{1}+\gamma_{2} \varphi_{2} \in H_{0}^{1}(\Gamma)\right\} & \\
W_{j} & :=\left\{\mathbf{q}_{j} \in H_{\text {div }}\left(\Omega_{j}\right) \mid \gamma_{\mathbf{n}_{j}} \mathbf{q}_{j} \in L^{2}(\Gamma)\right\} & \text { for } j=1,2, \\
W & :=W_{1} \oplus W_{2}, &
\end{aligned}
$$

which we need for the weak formulation of the reduced models. Let us define for all $\left(\varphi_{1}, \varphi_{2}\right) \in V$,

$$
\bar{\varphi}_{f}:=\frac{\gamma_{1} \varphi_{1}+\gamma_{2} \varphi_{2}}{2} \quad \text { and } \quad \delta_{x} \bar{\varphi}_{f}:=\frac{\gamma_{2} \varphi_{2}-\gamma_{1} \varphi_{1}}{2 \delta}
$$

and for all $\left(\mathbf{v}_{1}, \mathbf{v}_{2}\right) \in W$,

$$
\bar{v}_{f}:=\frac{\gamma_{\mathbf{n}_{1}} \mathbf{v}_{1}-\gamma_{\mathbf{n}_{2}} \mathbf{v}_{2}}{2} \quad \text { and } \quad \delta_{x} \bar{v}_{f}:=\frac{-\gamma_{\mathbf{n}_{2}} \mathbf{v}_{2}-\gamma_{\mathbf{n}_{1}} \mathbf{v}_{1}}{2 \delta} .
$$

The function space $V$ is complemented by the norm

$$
\left\|\left(\varphi_{1}, \varphi_{2}\right)\right\|_{V}=\left(\left(\sum_{j=1}^{2}\left\|\nabla \varphi_{j}\right\|_{L^{2}\left(\Omega_{j}\right)}^{2}\right)+2 \delta\left\|\partial_{y} \bar{\varphi}_{f}\right\|_{L^{2}(\Gamma)}^{2}+2 \delta\left\|\delta_{x} \bar{\varphi}_{f}\right\|_{L^{2}(\Gamma)}^{2}\right)^{\frac{1}{2}} .
$$


The $\delta$-weights in the norm are added in order to derive continuity and coercivity uniformly in $\delta$ for the bilinear form of the problem.

We can now multiply equations (5) by any $\varphi_{j} \in V_{j}$. Subsequent integration over $\Omega_{j}$, summation over $j=1,2$, and integration by parts, taking into account the definition of $\mathbf{q}_{j}$ in (6), yields

$$
\mathrm{a}_{\delta}\left(\left(u_{1}, u_{2}\right),\left(\varphi_{1}, \varphi_{2}\right)\right)=\sum_{j=1}^{2} \int_{\Omega_{j}} h_{j} \varphi_{j} \mathrm{~d} x \mathrm{~d} y,
$$

with the bilinear form on $V \times V$

$$
\begin{aligned}
& \operatorname{a}_{\delta}\left(\left(u_{1}, u_{2}\right),\left(\varphi_{1}, \varphi_{2}\right)\right)=2 \delta \int_{\Gamma}\left(\bar{\varphi}_{f} \delta_{x} \bar{q}_{f}+\bar{q}_{f} \delta_{x} \bar{\varphi}_{f}\right) \mathrm{d} y \\
& +\sum_{j=1}^{2} \int_{\Omega_{j}}\left(\left(\mathbf{A}_{j} \nabla-\frac{\mathbf{b}_{j}}{2}\right) u_{j} \cdot \nabla \varphi_{j}+\left(\frac{\mathbf{b}_{j}}{2} \cdot \nabla u_{j}\right) \varphi_{j}+\left(\eta_{j}-\operatorname{div} \frac{\mathbf{b}_{j}}{2}\right) u_{j} \varphi_{j}\right) \mathrm{d} x \mathrm{~d} y .
\end{aligned}
$$

By means of the coupling conditions (20), (21), we further obtain for the interfacial integral,

$$
\begin{aligned}
& \int_{\Gamma}\left(\bar{\varphi}_{f} \delta_{x} \bar{q}_{f}+\bar{q}_{f} \delta_{x} \bar{\varphi}_{f}\right) \mathrm{d} y=\int_{\Gamma}\left[\begin{array}{cc}
\left(\delta_{x} \bar{\varphi}_{f}\right. & \partial_{y} \bar{\varphi}_{f}
\end{array}\right) \mathbf{A}_{f}\left(\begin{array}{c}
\delta_{x} \bar{u}_{f} \\
\partial_{y} \bar{u}_{f}
\end{array}\right)+\eta_{f} \bar{u}_{f} \bar{\varphi}_{f} \\
& \left.-\frac{b_{1}^{f}}{2} \bar{u}_{f}\left(\delta_{x} \bar{\varphi}_{f}\right)+\frac{b_{1}^{f}}{2}\left(\delta_{x} \bar{u}_{f}\right) \bar{\varphi}_{f}+b_{2}^{f}\left(\partial_{y} \bar{u}_{f}\right) \bar{\varphi}_{f}\right] \mathrm{d} y .
\end{aligned}
$$

Therefore, the primal weak formulation of the reduced model with CC1 coupling conditions amounts to find $\left(u_{1}, u_{2}\right) \in V$, such that for all $\left(\varphi_{1}, \varphi_{2}\right) \in V$, one has $(23)$, (24).

Lemma 4.1 There exists a positive constant $C_{P}$, such that for all $\left(\varphi_{1}, \varphi_{2}\right) \in V$ we have the inequality

$$
\left(\sum_{j=1}^{2}\left(\left\|\varphi_{j}\right\|_{L^{2}\left(\Omega_{j}\right)}^{2}+2 \delta\left\|\bar{\varphi}_{f}\right\|_{L^{2}(\Gamma)}^{2}\right)^{\frac{1}{2}} \leq C_{P}\left\|\left(\varphi_{1}, \varphi_{2}\right)\right\|_{V}\right.
$$

Proof cf. [16] Proposition 1.2.1.

Remark 4.1 From the referenced proof of Lemma 4.1 it follows immediately that inequality (25) holds for all functions in $\left\{\left(\varphi_{1}, \varphi_{2}\right) \in H^{1}\left(\Omega_{1}\right) \oplus H^{1}\left(\Omega_{2}\right) \mid \gamma_{1} \varphi_{1}+\gamma_{2} \varphi_{2} \in H^{1}(\Gamma)\right\}$ with traces vanishing on a subset of the outer boundary of positive surface measure. The general requirement of the proof is that $\left(\varphi_{1}, \varphi_{2}\right)$ belongs to a closed subspace of $\left(H^{1}\left(\Omega_{1}\right) \oplus H^{1}\left(\Omega_{2}\right), \sum_{j=1}^{2}\|\cdot\|_{H^{1}\left(\Omega_{j}\right)}\right)$ for which $\|\cdot\|_{V}$ is a well defined norm.

Theorem 4.1 The bilinear form as associated with the reduced model (23), (24) is continuous and coercive uniformly with respect to $\delta$.

Proof Continuity: Let $\left(\varphi_{1}, \varphi_{2}\right) \in V$. From (24), there exists a positive constant $C$, independent of $\delta$, such that

$$
\begin{aligned}
& 2 \delta\left|\int_{\Gamma}\left(\bar{\varphi}_{f} \delta_{x} \bar{q}_{f}+\bar{q}_{f} \delta_{x} \bar{\varphi}_{f}\right) \mathrm{d} y\right| \\
& \quad \leq C\left(2 \delta\left\|\partial_{y} \bar{u}_{f}\right\|_{L^{2}(\Gamma)}^{2}+2 \delta\left\|\delta_{x} \bar{u}_{f}\right\|_{L^{2}(\Gamma)}^{2}+2 \delta\left\|\bar{u}_{f}\right\|_{L^{2}(\Gamma)}^{2}\right)^{\frac{1}{2}} \\
& \quad \cdot\left(2 \delta\left\|\partial_{y} \bar{\varphi}_{f}\right\|_{L^{2}(\Gamma)}^{2}+2 \delta\left\|\delta_{x} \bar{\varphi}_{f}\right\|_{L^{2}(\Gamma)}^{2}+2 \delta\left\|\bar{\varphi}_{f}\right\|_{L^{2}(\Gamma)}^{2}\right)^{\frac{1}{2}}
\end{aligned}
$$


Furthermore, there exists a positive constant $C$, such that for $j=1,2$,

$$
\begin{aligned}
& \left|\int_{\Omega_{j}}\left(\left(\mathbf{A}_{j} \nabla-\frac{\mathbf{b}_{j}}{2}\right) u_{j} \cdot \nabla \varphi_{j}+\left(\frac{\mathbf{b}_{j}}{2} \cdot \nabla u_{j}\right) \varphi_{j}+\left(\eta_{j}-\operatorname{div} \frac{\mathbf{b}_{j}}{2}\right) u_{j} \varphi_{j}\right) \mathrm{d} x \mathrm{~d} y\right| \\
& \leq C \sum_{j=1}^{2}\left(\left\|u_{j}\right\|_{L^{2}\left(\Omega_{j}\right)}+\left\|\nabla u_{j}\right\|_{L^{2}\left(\Omega_{j}\right)}\right)\left(\left\|\varphi_{j}\right\|_{L^{2}\left(\Omega_{j}\right)}+\left\|\nabla \varphi_{j}\right\|_{L^{2}\left(\Omega_{j}\right)}\right) .
\end{aligned}
$$

Hence, by (25) there exists a positive constant $C$, independent of $\delta$, such that

$$
\left|\mathrm{a}_{\delta}\left(\left(u_{1}, u_{2}\right),\left(\varphi_{1}, \varphi_{2}\right)\right)\right| \leq C\left\|\left(u_{1}, u_{2}\right)\right\|_{V}\left\|\left(\varphi_{1}, \varphi_{2}\right)\right\|_{V}
$$

Coercivity: Let us first note that, from $\bar{\varphi}_{f}=\bar{u}_{f}$ in (24), we obtain

$$
\begin{aligned}
& 2 \delta \int_{\Gamma}\left(\bar{u}_{f} \delta_{x} \bar{q}_{f}+\bar{q}_{f} \delta_{x} \bar{u}_{f}\right) \mathrm{d} y \\
& =2 \delta \int_{\Gamma}\left(\begin{array}{ll}
\delta_{x} \bar{u}_{f} & \partial_{y} \bar{u}_{f}
\end{array}\right) \mathbf{A}_{f}\left(\begin{array}{c}
\delta_{x} \bar{u}_{f} \\
\partial_{y} \bar{u}_{f}
\end{array}\right) d y+2 \delta \int_{\Gamma} \eta_{f}\left(\bar{u}_{f}\right)^{2} d y \\
& \geq \lambda_{\min }\left(\mathbf{A}_{f}\right) 2 \delta\left(\left\|\delta_{x} \bar{u}_{f}\right\|_{L^{2}(\Gamma)}^{2}+\left\|\partial_{y} \bar{u}_{f}\right\|_{L^{2}(\Gamma)}^{2}\right)+2 \delta \eta_{f}\left\|\bar{u}_{f}\right\|_{L^{2}(\Gamma)}^{2} .
\end{aligned}
$$

Furthermore, for $j=1,2$,

$$
\begin{aligned}
& \int_{\Omega_{j}}\left(\left(\mathbf{A}_{j} \nabla-\frac{\mathbf{b}_{j}}{2}\right) u_{j} \cdot \nabla u_{j}+\left(\frac{\mathbf{b}_{j}}{2} \cdot \nabla u_{j}\right) u_{j}+\left(\eta_{j}-\operatorname{div} \frac{\mathbf{b}_{j}}{2}\right) u_{j}^{2}\right) \mathrm{d} x \mathrm{~d} y \\
& \geq \sum_{j=1}^{2}\left(\left(\eta_{j}-\operatorname{div} \frac{\mathbf{b}_{j}}{2}\right)\left\|u_{j}\right\|_{L^{2}\left(\Omega_{j}\right)}^{2}+\lambda_{\min }\left(\mathbf{A}_{j}\right)\left\|\nabla u_{j}\right\|_{L^{2}\left(\Omega_{j}\right)}^{2} .\right.
\end{aligned}
$$

Inserting $\left(u_{1}, u_{2}\right) \in V$ as a test function in the bilinear form of the variational problem (23), (24) immediately yields its coercivity, with a constant $C$ independent of $\delta$

$$
\mathrm{a}_{\delta}\left(\left(u_{1}, u_{2}\right),\left(u_{1}, u_{2}\right)\right) \geq C\left\|\left(u_{1}, u_{2}\right)\right\|_{V}^{2} .
$$

Remark 4.2 The coercivity for the reduced problem with CCO coupling condition is immediate. However, the reduced problems with higher than next-to-leading-order (CC1) coupling conditions are not coercive, in general. In the corresponding calculations for the next-to-next-to-leadingorder coupling conditions, a term related to the normal fracture advection coefficient can not be controlled.

\section{$5 \quad$ Fracture reconstruction for reduced models}

In many applications, the fracture unknown $u_{f}$ is of interest. Substituting (13) into equation (7) yields an expression for $\hat{u}_{f}$ in terms of $\hat{\gamma}_{1} \hat{u}_{1}$ and $\hat{\gamma}_{1} \hat{u}_{2}$,

$$
\hat{u}_{f}(x, k)=\frac{\left(\hat{\gamma}_{2} \hat{u}_{2}(\delta, k) e^{-\delta \lambda_{2}}-\hat{\gamma}_{1} \hat{u}_{1}(-\delta, k) e^{\delta \lambda_{2}}\right) e^{\lambda_{1} x}+\left(\hat{\gamma}_{1} \hat{u}_{1}(-\delta, k) e^{\delta \lambda_{1}}-\hat{\gamma}_{2} \hat{u}_{2}(\delta, k) e^{-\delta \lambda_{1}}\right) e^{\lambda_{2} x}}{2 \sinh (2 s \delta)},
$$

which can be used to recover information on $u_{f}$. We will give now some examples. 
Approximation for $u_{f}(0, y)$ : we start with

$$
\begin{aligned}
\hat{u}_{f}(0, k) & =\frac{\hat{\gamma}_{2} \hat{u}_{2} e^{-\delta \lambda_{2}}-\hat{\gamma}_{1} \hat{u}_{1} e^{\delta \lambda_{2}}+\hat{\gamma}_{1} \hat{u}_{1} e^{\delta \lambda_{1}}-\hat{\gamma}_{2} \hat{u}_{2} e^{-\delta \lambda_{1}}}{2 \sinh (2 s \delta)}=\frac{\hat{\gamma}_{1} \hat{u}_{1} e^{\delta r}+\hat{\gamma}_{2} \hat{u}_{2} e^{-\delta r}}{2 \cosh (s \delta)} \\
& =\frac{1}{2}\left(\hat{\gamma}_{1} \hat{u}_{1}+\hat{\gamma}_{2} \hat{u}_{2}\right)-\frac{\delta}{4 a_{11}}\left(\left(a_{12}+a_{21}\right) i k-b_{1}\right)\left(\hat{\gamma}_{1} \hat{u}_{1}-\hat{\gamma}_{2} \hat{u}_{2}\right) \\
& -\frac{\delta^{2}}{4 a_{11}}\left(a_{22} k^{2}+b_{2} i k+\eta\right)\left(\hat{\gamma}_{1} \hat{u}_{1}+\hat{\gamma}_{2} \hat{u}_{2}\right)+\mathcal{O}\left(\delta^{3}\right) .
\end{aligned}
$$

Then, truncating at a given order and performing an inverse Fourier transform (18) gives rise to the following definitions:

1. Truncation after the leading-order term:

$$
u_{f}^{\text {red,0 }}:=\frac{1}{2}\left(\gamma_{1} u_{1}^{\text {red }}+\gamma_{2} u_{2}^{\text {red }}\right) .
$$

2. Truncation after the next-to-leading-order term:

$$
u_{f}^{\mathrm{red}, 1}:=\frac{1}{2}\left(\gamma_{1} u_{1}^{\mathrm{red}}+\gamma_{2} u_{2}^{\mathrm{red}}\right)-\frac{\delta}{4 a_{11}}\left(\left(a_{12}+a_{21}\right) \partial_{y}-b_{1}\right)\left(\gamma_{1} u_{1}^{\mathrm{red}}-\gamma_{2} u_{2}^{\mathrm{red}}\right) .
$$

3. Truncation after the next-to-next-to-leading-order term:

$$
\begin{aligned}
u_{f}^{\mathrm{red}, 2} & :=\frac{1}{2}\left(\gamma_{1} u_{1}^{\mathrm{red}}+\gamma_{2} u_{2}^{\mathrm{red}}\right)-\frac{\delta}{4 a_{11}}\left(\left(a_{12}+a_{21}\right) \partial_{y}-b_{1}\right)\left(\gamma_{1} u_{1}^{\mathrm{red}}-\gamma_{2} u_{2}^{\mathrm{red}}\right) \\
& +\frac{\delta^{2}}{4 a_{11}}\left(a_{22} \partial_{y y}-b_{2} \partial_{y}-\eta\right)\left(\gamma_{1} u_{1}^{\mathrm{red}}+\gamma_{2} u_{2}^{\mathrm{red}}\right) .
\end{aligned}
$$

Approximation for $U_{f}(y):=\frac{1}{2 \delta} \int_{-\delta}^{\delta} u_{f}(x, y) \mathrm{d} x$ : let us first calculate the Fourier coefficients for $U_{f}(y)$,

$$
\begin{aligned}
\hat{U}_{f}(k)= & \frac{1}{2 \delta} \int_{-\delta}^{\delta} \hat{u}_{f}(x, k) \mathrm{d} x \\
= & \frac{-e^{-2 \delta(r+s)}}{\left.8 \delta\left(r^{2}-s^{2}\right) \sinh (\delta s) \cosh (\delta s)\right)} \\
& \cdot\left(-2 e^{(4 r+2 s) \delta} s \hat{\gamma}_{1} \hat{u}_{1}+\left(\left(\hat{\gamma}_{1} \hat{u}_{1}+\hat{\gamma}_{2} \hat{u}_{2}\right) s+\left(\hat{\gamma}_{1} \hat{u}_{1}-\hat{\gamma}_{2} \hat{u}_{2}\right) r\right) e^{2 \delta(r+2 s)}\right. \\
& \left.+\left(\left(\hat{\gamma}_{1} \hat{u}_{1}+\hat{\gamma}_{2} \hat{u}_{2}\right) s-\left(\hat{\gamma}_{1} \hat{u}_{1}-\hat{\gamma}_{2} \hat{u}_{2}\right) r\right) e^{2 \delta r}-2 s \hat{\gamma}_{2} \hat{u}_{2} e^{2 \delta s}\right) \\
= & \frac{1}{2}\left(\hat{\gamma}_{1} \hat{u}_{1}+\hat{\gamma}_{2} \hat{u}_{2}\right)-\frac{\delta}{6 a_{11}}\left(\left(a_{12}+a_{21}\right) i k-b_{1}\right)\left(\hat{\gamma}_{1} \hat{u}_{1}-\hat{\gamma}_{2} \hat{u}_{2}\right) \\
& -\frac{\delta^{2}}{6 a_{11}}\left(a_{22} k^{2}+b_{2} i k+\eta\right)\left(\hat{\gamma}_{1} \hat{u}_{1}+\hat{\gamma}_{2} \hat{u}_{2}\right)+\mathcal{O}\left(\delta^{3}\right) .
\end{aligned}
$$

Truncating at a given order and using an inverse Fourier transform gives rise to the following definitions:

1. Truncation after the leading-order term:

$$
U_{f}^{\mathrm{red}, 0}:=\frac{1}{2}\left(\gamma_{1} u_{1}^{\mathrm{red}}+\gamma_{2} u_{2}^{\mathrm{red}}\right)
$$

2. Truncation after the next-to-leading-order term:

$$
U_{f}^{\mathrm{red}, 1}:=\frac{1}{2}\left(\gamma_{1} u_{1}^{\mathrm{red}}+\gamma_{2} u_{2}^{\mathrm{red}}\right)-\frac{\delta}{6 a_{11}}\left(\left(a_{12}+a_{21}\right) \partial_{y}-b_{1}\right)\left(\gamma_{1} u_{1}^{\mathrm{red}}-\gamma_{2} u_{2}^{\mathrm{red}}\right) .
$$


3. Truncation after the next-to-next-to-leading-order term:

$$
\begin{aligned}
U_{f}^{\mathrm{red}, 2} & :=\frac{1}{2}\left(\gamma_{1} u_{1}^{\mathrm{red}}+\gamma_{2} u_{2}^{\mathrm{red}}\right)-\frac{\delta}{6 a_{11}}\left(\left(a_{12}+a_{21}\right) \partial_{y}-b_{1}\right)\left(\gamma_{1} u_{1}^{\mathrm{red}}-\gamma_{2} u_{2}^{\mathrm{red}}\right) \\
& +\frac{\delta^{2}}{6 a_{11}}\left(a_{22} \partial_{y y}-b_{2} \partial_{y}-\eta\right)\left(\gamma_{1} u_{1}^{\mathrm{red}}+\gamma_{2} u_{2}^{\mathrm{red}}\right) .
\end{aligned}
$$

\section{Comparison to the literature}

DFM models are a tool for the simulation of flow through fractured porous media, where the governing equations are mass conservation and Darcy's law. The approach illustrated above covers more general problems, and in order to compare our models to existing ones from the literature, we now let

$$
\mathbf{b}:=0, \quad \eta:=0, \quad \text { and } \quad \mathbf{A}:=\left(\begin{array}{cc}
a_{11} & 0 \\
0 & a_{22}
\end{array}\right) .
$$

As outlined in [19], a PDE on the dimensionally reduced fracture $\Gamma$ is derived by integrating the mass conservation equation over the fracture width,

$$
0=\int_{-\delta}^{\delta} \operatorname{div} \mathbf{q}_{f} \mathrm{~d} x=\gamma_{\mathbf{n}_{f, 2}} \mathbf{q}_{f}+\gamma_{\mathbf{n}_{f, 1}} \mathbf{q}_{f}+\partial_{y} \int_{-\delta}^{\delta} \mathbf{q}_{f} \cdot \boldsymbol{\tau} \mathrm{d} x
$$

with $\boldsymbol{\tau}$ being the unit vector tangetial to $\Gamma$. Hence, by means of Darcy's law and normal flux continuity, we get

$$
-\gamma_{\mathbf{n}_{2}} \mathbf{q}_{2}-\gamma_{\mathbf{n}_{1}} \mathbf{q}_{1}+2 \delta a_{22} \partial_{y}^{2} U_{f}=0
$$

where $U_{f}:=\frac{1}{2 \delta} \int_{-\delta}^{\delta} u_{f} \mathrm{~d} x$ is the fracture unknown. Then, one typically derives one of the reduced order matrix-fracture $(m f)$ coupling conditions by integrating Darcy's law over the fracture width,

$$
\int_{-\delta}^{\delta} \mathbf{q}_{f} \cdot \mathbf{n} \mathrm{d} x=a_{11}\left(\gamma_{f, 2} u_{f}-\gamma_{f, 1} u_{f}\right)=a_{11}\left(\gamma_{2} u_{2}-\gamma_{1} u_{1}\right)
$$

and by using the trapezoidal approximation

$$
\int_{-\delta}^{\delta} \mathbf{q}_{f} \cdot \mathbf{n} \mathrm{d} x \approx 2 \delta \frac{\gamma_{\mathbf{n}_{f, 2}} \mathbf{q}_{f}-\gamma_{\mathbf{n}_{f, 1}} \mathbf{q}_{f}}{2}=\delta\left(\gamma_{\mathbf{n}_{1}} \mathbf{q}_{1}-\gamma_{\mathbf{n}_{2}} \mathbf{q}_{2}\right),
$$

which yields the $m f$ coupling condition

$$
\delta\left(\gamma_{\mathbf{n}_{1}} \mathbf{q}_{1}-\gamma_{\mathbf{n}_{2}} \mathbf{q}_{2}\right) \approx a_{11}\left(\gamma_{2} u_{2}-\gamma_{1} u_{1}\right) .
$$

In order to provide the second $m f$ coupling condition, the authors propose in [19] a family of approximations for $U_{f}$, parametrized by $\xi \in\left[\frac{1}{2}, 1\right]$,

$$
U_{f} \approx \frac{\gamma_{2} u_{2}+\gamma_{1} u_{1}}{2}+\frac{2 \xi-1}{2} \frac{\delta}{a_{11}}\left(\gamma_{\mathbf{n}_{1}} \mathbf{q}_{1}+\gamma_{\mathbf{n}_{2}} \mathbf{q}_{2}\right) .
$$

The corresponding reduced order model amounts to find $u_{j}^{\xi}, \mathbf{q}_{j}^{\xi}, U_{f}^{\xi}$, such that

$$
\begin{aligned}
-\operatorname{div} \mathbf{q}_{j}^{\xi}+\frac{\mathbf{b}_{j}}{2} \cdot \nabla u_{j}^{\xi}+\left(\eta_{j}-\operatorname{div} \frac{\mathbf{b}_{j}}{2}\right) u_{j}^{\xi} & =h_{j} & & \text { in } \Omega_{j}, j=1,2, \\
\mathbf{q}_{j}^{\xi} & =\left(\mathbf{A}_{j} \nabla-\frac{\mathbf{b}_{j}}{2}\right) u_{j}^{\xi} & & \text { in } \Omega_{j}, j=1,2, \\
2 \delta a_{22} \partial_{y}^{2} U_{f}^{\xi} & =\gamma_{\mathbf{n}_{1}} \mathbf{q}_{1}^{\xi}+\gamma_{\mathbf{n}_{2}} \mathbf{q}_{2}^{\xi} & & \text { on } \Gamma, \\
\delta\left(\gamma_{\mathbf{n}_{1}} \mathbf{q}_{1}^{\xi}-\gamma_{\mathbf{n}_{2}} \mathbf{q}_{2}^{\xi}\right) & =a_{11}\left(\gamma_{2} u_{2}^{\xi}-\gamma_{1} u_{1}^{\xi}\right) & & \text { on } \Gamma, \\
\frac{\gamma_{2} u_{2}^{\xi}+\gamma_{1} u_{1}^{\xi}}{2}+\frac{2 \xi-1}{2} \frac{\delta}{a_{11}}\left(\gamma_{\mathbf{n}_{1}} \mathbf{q}_{1}^{\xi}+\gamma_{\mathbf{n}_{2}} \mathbf{q}_{2}^{\xi}\right) & =U_{f}^{\xi} & & \text { on } \Gamma .
\end{aligned}
$$


Theorem 6.1 For $\xi=\frac{1}{2}$, the model (39)-(43) is equivalent to the model (5), (6), (20), (21), (32), for the diffusion equation with diagonal matrix $\mathbf{A}_{f}$ inside the fracture.

Proof The statement of the theorem follows by substituting equation (43) into (41).

Writing (16), (17) for the diffusion model with diagonal matrix $A$, we observe that the asymptotic behavior of the exact coupling conditions depends only on the asymptotic behavior of the ratio $\frac{\delta}{a_{11}}$ and of the product $\delta a_{22}$. We call these two characteristic quantities the fracture resistivity and fracture conductivity. In [22], a rigorous asymptotic analysis for the Laplace equation is performed, with the focus on the solution in the limit $\delta=0$. In this context, coupling conditions (at $x= \pm 0$ ) are derived, for the cases $\frac{\delta}{a_{11}} \rightarrow \alpha \in \mathbb{R}, \frac{\delta}{a_{11}} \rightarrow \infty, \frac{\delta}{a_{11}} \rightarrow 0$, provided $a_{11} \rightarrow 0$, which turn out to correspond to the coupling conditions which we derive by means of truncating (16), (17) at order $\delta^{0}$ (with $\nu:=a_{11}=a_{22}$ for isotropic diffusion).

1. Case $\frac{\delta}{\nu} \rightarrow \alpha \in \mathbb{R}$ (note that this implies $\delta \nu \rightarrow 0$ ):

$$
\gamma_{\mathbf{n}_{1}} \mathbf{q}_{1}+\gamma_{\mathbf{n}_{2}} \mathbf{q}_{2}=0 \quad \text { and } \quad \gamma_{2} u_{2}-\gamma_{1} u_{1}=\alpha\left(\gamma_{\mathbf{n}_{1}} \mathbf{q}_{1}-\gamma_{\mathbf{n}_{2}} \mathbf{q}_{2}\right) \text {. }
$$

2. Case $\frac{\delta}{\nu} \rightarrow \infty$ (note that this implies $\left.\delta \nu \rightarrow 0\right)$ :

$$
\gamma_{\mathbf{n}_{1}} \mathbf{q}_{1}+\gamma_{\mathbf{n}_{2}} \mathbf{q}_{2}=0 \quad \text { and } \quad \gamma_{\mathbf{n}_{1}} \mathbf{q}_{1}-\gamma_{\mathbf{n}_{2}} \mathbf{q}_{2}=0
$$

3. Case $\frac{\delta}{\nu} \rightarrow 0$ and $\delta \nu \rightarrow 0$ corresponds to (19).

We can now complete this study by considering the cases $\delta \nu \rightarrow \alpha \in \mathbb{R}$ or $\delta \nu \rightarrow \infty$ (which both imply $\left.\frac{\delta}{\nu} \rightarrow 0\right)$. We obtain

4. Case $\delta \nu \rightarrow \alpha \in \mathbb{R}:$

$$
\gamma_{\mathbf{n}_{1}} \mathbf{q}_{1}+\gamma_{\mathbf{n}_{2}} \mathbf{q}_{2}=\alpha \partial_{y y}\left(u_{1}+u_{2}\right) \quad \text { and } \quad \gamma_{2} u_{2}-\gamma_{1} u_{1}=0
$$

5. Case $\delta \nu \rightarrow \infty:$

$$
\partial_{y y}\left(\gamma_{1} u_{1}+\gamma_{2} u_{2}\right)=0 \quad \text { and } \quad \gamma_{2} u_{2}-\gamma_{1} u_{1}=0
$$

\section{Error estimates for the reduced models}

In this section, we will derive an error estimate in the $H^{1}$-norm for the reduced order solution in the bulk (matrix) domains and error estimates in the $H^{\frac{1}{2}}$-norm for the reduced order reconstructed fracture solutions. For simplicity, we restrict ourselves to the anisotropic diffusion equation with diagonal tensor inside the fracture, as in (35), and to the isotropic diffusion equation in the matrix. Furthermore, the underlying geometry is set to $\Omega_{1}=\left(-L_{1},-\delta\right) \times \Gamma$, $\Omega_{f}=(-\delta, \delta) \times \Gamma, \Omega_{1}=\left(\delta, L_{2}\right) \times \Gamma$, with $\delta<L_{1}, L_{2}<\infty$ and $\Gamma=\mathbb{R}$, in order to allow for the use of Fourier transforms w.r.t. the $y$-coordinate (similar results could also be obtained on bounded domains using Fourier series).

In this setting, the model solved on the full domain consists of the Poisson equation in mixed formulation in the matrix,

$$
\begin{aligned}
-\operatorname{divq}_{j} & =h_{j} & & \text { in } \Omega_{j}, \\
\mathbf{q}_{j} & =\nabla u_{j} & & \text { in } \Omega_{j}, \\
\gamma_{\partial \Omega} u_{j} & =0 & & \text { on } \partial \Omega_{j} \backslash \Gamma_{j},
\end{aligned}
$$

$j \in\{1,2\}$, and an anisotropic diffusion model inside the fracture,

$$
\begin{aligned}
-\operatorname{div} \mathbf{q}_{f} & =0 & & \text { in } \Omega_{f}, \\
\mathbf{q}_{j} & =\left(a_{11} \partial_{x x}+a_{22} \partial_{y y}\right) u_{f} & & \text { in } \Omega_{f},
\end{aligned}
$$


together with the matrix-fracture coupling conditions

$$
\begin{aligned}
\gamma_{j} u_{j} & =\gamma_{f, j} u_{f} & & \text { on } \Gamma_{j}, \\
\gamma_{\mathbf{n}_{j}} \mathbf{q}_{j} & =-\gamma_{\mathbf{n}_{f, j}} \mathbf{q}_{f} & & \text { on } \Gamma_{j} .
\end{aligned}
$$

The reduced order model consists of the Poisson equation in mixed formulation in the matrix,

$$
\begin{aligned}
-\operatorname{div} \mathbf{q}_{j}^{\text {red }} & =h_{j} & & \text { in } \Omega_{j}, \\
\mathbf{q}_{j}^{\text {red }} & =\nabla u_{j}^{\text {red }} & & \text { in } \Omega_{j}, \\
\gamma_{\partial \Omega} u_{j}^{\text {red }} & =0 & & \text { on } \partial \Omega_{j} \backslash \Gamma_{j},
\end{aligned}
$$

$j \in\{1,2\}$, together with CC1 coupling conditions (see (20), (21)),

$$
\begin{aligned}
\gamma_{\mathbf{n}_{2}} \mathbf{q}_{2}^{\text {red }}+\gamma_{\mathbf{n}_{1}} \mathbf{q}_{1}^{\text {red }} & =\delta a_{22} \partial_{y y}\left(\gamma_{1} u_{1}^{\text {red }}+\gamma_{2} u_{2}^{\text {red }}\right), \\
-\gamma_{\mathbf{n}_{2}} \mathbf{q}_{2}^{\text {red }}+\gamma_{\mathbf{n}_{1}} \mathbf{q}_{1}^{\text {red }} & =\frac{a_{11}}{\delta}\left(\gamma_{2} u_{2}^{\text {red }}-\gamma_{1} u_{1}^{\text {red }}\right) .
\end{aligned}
$$

Theorem 7.1 (Matrix error estimate) Let $\mathbf{A}_{f}$ be diagonal, $\mathbf{A}_{1}=\mathbf{A}_{2}=\mathbf{I}, \mathbf{b}_{1}=\mathbf{b}_{2}=\mathbf{b}_{f}=$ 0 and $\eta_{1}=\eta_{2}=\eta_{f}=0$. Let $\left\{u_{1}, u_{2}, u_{f}\right\}$ be solution to (1)-(4) and $\left\{u_{1}^{\text {red }}, u_{2}^{\text {red }}\right\}$ be solution to (5), (6), (20) and (21). Then there exists a constant $C>0$ independent of $\delta$, such that for $j=1,2$ we have the estimate

$$
\left\|u_{j}-u_{j}^{\mathrm{red}}\right\|_{H^{1}\left(\Omega_{j}\right)} \leq C \delta^{3}\left(\left\|u_{1}\right\|_{H^{4}\left(\Omega_{1}\right)}+\left\|u_{2}\right\|_{H^{4}\left(\Omega_{2}\right)}+\left\|h_{1}\right\|_{H^{3}\left(\Omega_{1}\right)}+\left\|h_{2}\right\|_{H^{3}\left(\Omega_{2}\right)}\right) .
$$

Proof We want to express the normal traces of the matrix fluxes in terms of the $h_{j}$ and the traces of $u_{j}$ on $\Gamma_{j}, j \in\{1,2\}$. To do so, we introduce the Steklov-Poincaré operators

$$
\begin{aligned}
\mathcal{S}_{j}: H^{\frac{1}{2}}\left(\Gamma_{j}\right) & \longrightarrow H^{-\frac{1}{2}}\left(\Gamma_{j}\right) \\
g_{j} & \mapsto \gamma_{\mathbf{n}_{j}}\left(\nabla v_{j}\right),
\end{aligned}
$$

where $v_{j}$ satisfies the equations

$$
\begin{array}{rlrl}
-\Delta v_{j} & =0 & & \text { in } \Omega_{j}, \\
\gamma_{j} v_{j}=g_{j} & & \text { on } \Gamma_{j}, \\
\gamma_{\partial \Omega} v_{j} & =0 & & \text { on } \partial \Omega \cap \partial \Omega_{j} .
\end{array}
$$

To account for the source term, we also introduce the operators

$$
\begin{aligned}
\mathcal{R}_{j}: L^{2}\left(\Omega_{j}\right) & \longrightarrow H^{-\frac{1}{2}}\left(\Gamma_{j}\right) \\
h_{j} & \mapsto \gamma_{\mathbf{n}_{j}}\left(\nabla w_{j}\right),
\end{aligned}
$$

where $w_{j}$ satisfies the equations

$$
\begin{aligned}
-\Delta w_{j} & =h_{j} & & \text { in } \Omega_{j}, \\
\gamma_{j} w_{j} & =0 & & \text { on } \Gamma_{j}, \\
\gamma_{\partial \Omega} w_{j} & =0 & & \text { on } \partial \Omega \cap \partial \Omega_{j} .
\end{aligned}
$$

Then, from the superposition principle for linear differential equations, we have

$$
\begin{aligned}
\gamma_{\mathbf{n}_{j}} \mathbf{q}_{j} & =\mathcal{S}_{j}\left(\gamma_{j} u_{j}\right)+\mathcal{R}_{j}\left(h_{j}\right), \\
\gamma_{\mathbf{n}_{j}} \mathbf{q}_{j}^{\text {red }} & =\mathcal{S}_{j}\left(\gamma_{j} u_{j}^{\text {red }}\right)+\mathcal{R}_{j}\left(h_{j}\right) .
\end{aligned}
$$

In a first step, we will derive an error estimate for the traces on $\Gamma_{j}$ of the reduced order solutions in Fourier space. As shown in Section 3, the exact problem in Fourier space, after 
the elimination of the fracture unknown by means of a continuous Schur complement, can be written as

$$
\begin{array}{ll}
\left(k^{2}-\partial_{x x}\right) \hat{u}_{1}=\hat{h}_{1} & \text { in }\left(-L_{1},-\delta\right), \forall k \in \mathbb{R}, \\
\left(k^{2}-\partial_{x x}\right) \hat{u}_{2}=\hat{h}_{2} & \text { in }\left(\delta, L_{2}\right), \forall k \in \mathbb{R},
\end{array}
$$

together with the coupling conditions

$$
\begin{aligned}
& \hat{s}_{1} \hat{\gamma}_{1} \hat{u}_{1}+\hat{s}_{2} \hat{\gamma}_{2} \hat{u}_{2}+\widehat{\mathcal{R}_{1}\left(h_{1}\right)}+\widehat{\mathcal{R}_{2}\left(h_{2}\right)}=-\hat{f}^{\mathrm{ex}}\left(\hat{\gamma}_{1} \hat{u}_{1}+\hat{\gamma}_{2} \hat{u}_{2}\right), \\
& \hat{s}_{1} \hat{\gamma}_{1} \hat{u}_{1}-\hat{s}_{2} \hat{\gamma}_{2} \hat{u}_{2}+\widehat{\mathcal{R}_{1}\left(h_{1}\right)}-\widehat{\mathcal{R}_{2}\left(h_{2}\right)}=\hat{g}^{\mathrm{ex}}\left(\hat{\gamma}_{2} \hat{u}_{2}-\hat{\gamma}_{1} \hat{u}_{1}\right),
\end{aligned}
$$

where we have introduced the terms

$$
\hat{f}^{\mathrm{ex}}:=a_{11} \sqrt{\frac{a_{22}}{a_{11}} k^{2}} \tanh \left(\delta \sqrt{\frac{a_{22}}{a_{11}} k^{2}}\right), \quad \hat{g}^{\mathrm{ex}}:=\frac{a_{11} \sqrt{\frac{a_{22}}{a_{11}} k^{2}}}{\tanh \left(\delta \sqrt{\frac{a_{22}}{a_{11}} k^{2}}\right)},
$$

and where

$$
\hat{s}_{j} \hat{\gamma}_{j} \hat{u}_{j}:=\widehat{\mathcal{S}_{j}\left(u_{j}\right)}=|k| \operatorname{coth}\left(|k|\left(L_{j}-\delta\right)\right) \hat{\gamma}_{j} \hat{u}_{j}
$$

The property of the Steklov-Poincaré operators to reduce to a scaling factor in Fourier space will be used in what follows.

The reduced order model in Fourier space can be written in the form

$$
\left(k^{2}-\partial_{x x}\right) \hat{u}_{j}^{\text {red }}=\hat{h}_{j} \quad \text { in }( \pm L, \pm \delta), \forall k \in \mathbb{R},
$$

together with the coupling conditions

$$
\begin{aligned}
& \hat{s}_{1} \hat{\gamma}_{1} \hat{u}_{1}^{\mathrm{red}}+\hat{s}_{2} \hat{\gamma}_{2} \hat{u}_{2}^{\mathrm{red}}+\widehat{\mathcal{R}_{1}\left(h_{1}\right)}+\widehat{\mathcal{R}_{2}\left(h_{2}\right)}=-\hat{f}^{\mathrm{red}}\left(\hat{\gamma}_{1} \hat{u}_{1}^{\mathrm{red}}+\hat{\gamma}_{2} \hat{u}_{2}^{\mathrm{red}}\right), \\
& \hat{s}_{1} \hat{\gamma}_{1} \hat{u}_{1}^{\mathrm{red}}-\hat{s}_{2} \hat{\gamma}_{2} \hat{u}_{2}^{\mathrm{red}}+\widehat{\mathcal{R}_{1}\left(h_{1}\right)}-\widehat{\mathcal{R}_{2}\left(h_{2}\right)}=\hat{g}^{\mathrm{red}}\left(\hat{\gamma}_{2} \hat{u}_{2}^{\mathrm{red}}-\hat{\gamma}_{1} \hat{u}_{1}^{\mathrm{red}}\right),
\end{aligned}
$$

where we have introduced the terms

$$
\hat{f}^{\mathrm{red}}:=\delta a_{22} k^{2}, \quad \hat{g}^{\mathrm{red}}:=\frac{a_{11}}{\delta} .
$$

Combining equations (45), (46), (47) and (48) yields the expressions for the error of the traces on $\Gamma_{j}$ in Fourier space,

$$
\begin{aligned}
\hat{\gamma}_{1} \hat{e}_{1} & :=\hat{\gamma}_{1} \hat{u}_{1}-\hat{\gamma}_{1} \hat{u}_{1}^{\text {red }} \\
= & \frac{-\left(\hat{s}_{2}+\hat{g}^{\text {red }}\right)\left(\hat{f}^{\mathrm{ex}}-\hat{f}^{\mathrm{red}}\right)\left(\hat{\gamma}_{2} \hat{u}_{2}+\hat{\gamma}_{1} \hat{u}_{1}\right)+\left(\hat{s}_{2}+\hat{f}^{\mathrm{red}}\right)\left(\hat{g}^{\mathrm{ex}}-\hat{g}^{\mathrm{red}}\right)\left(\hat{\gamma}_{2} \hat{u}_{2}-\hat{\gamma}_{1} \hat{u}_{1}\right)}{\left(\hat{s}_{2}+\hat{g}^{\mathrm{red}}\right)\left(\hat{s}_{1}+\hat{f}^{\mathrm{red}}\right)+\left(\hat{s}_{1}+\hat{g}^{\mathrm{red}}\right)\left(\hat{s}_{2}+\hat{f}^{\mathrm{red}}\right)} \\
\hat{\gamma}_{2} \hat{e}_{2}:=\hat{\gamma}_{2} \hat{u}_{2}-\hat{\gamma}_{2} \hat{u}_{2}^{\text {red }} & \\
= & \frac{-\left(\hat{s}_{1}+\hat{g}^{\mathrm{red}}\right)\left(\hat{f}^{\mathrm{ex}}-\hat{f}^{\mathrm{red}}\right)\left(\hat{\gamma}_{2} \hat{u}_{2}+\hat{\gamma}_{1} \hat{u}_{1}\right)-\left(\hat{s}_{1}+\hat{f}^{\mathrm{red}}\right)\left(\hat{g}^{\mathrm{ex}}-\hat{g}^{\mathrm{red}}\right)\left(\hat{\gamma}_{2} \hat{u}_{2}-\hat{\gamma}_{1} \hat{u}_{1}\right)}{\left(\hat{s}_{2}+\hat{g}^{\mathrm{red}}\right)\left(\hat{s}_{1}+\hat{f}^{\mathrm{red}}\right)+\left(\hat{s}_{1}+\hat{g}^{\mathrm{red}}\right)\left(\hat{s}_{2}+\hat{f}^{\mathrm{red}}\right)} .
\end{aligned}
$$

We will now give estimates for these errors in the sharpest possible order in $\delta$. First, let us estimate the coefficient in front of the sum $\left(\hat{\gamma}_{2} \hat{u}_{2}+\hat{\gamma}_{1} \hat{u}_{1}\right)$. We have

$$
\begin{aligned}
\left|\hat{f}^{\mathrm{ex}}-\hat{f}^{\mathrm{red}}\right| & =|k| \sqrt{a_{11} a_{22}}\left|\tanh \left(|k| \delta \sqrt{\frac{a_{22}}{a_{11}}}\right)-\right| k\left|\delta \sqrt{\frac{a_{22}}{a_{11}}}\right| \\
& \leq|k| \sqrt{a_{11} a_{22}} \sup _{z \in \mathbb{R}}\left|\frac{\tanh z-z}{z^{3}}\right||k|^{3} \delta^{3}\left(\frac{a_{22}}{a_{11}}\right)^{\frac{3}{2}}
\end{aligned}
$$


and, for $j \in\{1,2\}$, using the notation $\{j+1\}=2$ for $j=1$ and $\{j+1\}=1$ for $j=2$,

$$
0<\frac{\left(\hat{s}_{j}+\hat{g}^{\mathrm{red}}\right)}{\left(\hat{s}_{2}+\hat{g}^{\mathrm{red}}\right)\left(\hat{s}_{1}+\hat{f}^{\mathrm{red}}\right)+\left(\hat{s}_{1}+\hat{g}^{\mathrm{red}}\right)\left(\hat{s}_{2}+\hat{f}^{\mathrm{red}}\right)} \leq \frac{1}{\hat{s}_{\{j+1\}}+\hat{f}^{\mathrm{red}}} \leq \frac{1}{\hat{s}_{\{j+1\}}} \leq \frac{1}{|k|},
$$

where we have used the positivity of the occurring coefficients, and the estimate

$$
|k| \leq|k| \operatorname{coth}(|k|(L-\delta)) \leq|k|+\frac{1}{L-\delta} .
$$

Now, let us estimate the coefficient in front of the difference $\left(\hat{\gamma}_{2} \hat{u}_{2}-\hat{\gamma}_{1} \hat{u}_{1}\right)$. We have

$$
\left|\hat{g}^{\mathrm{ex}}-\hat{g}^{\mathrm{red}}\right|=\frac{a_{11}}{\delta}\left|\frac{|k| \delta \sqrt{\frac{a_{22}}{a_{11}}}}{\tanh \left(|k| \delta \sqrt{\frac{a_{22}}{a_{11}}}\right)}-1\right| \leq \frac{a_{11}}{\delta} \sup _{z \in \mathbb{R}}\left|\frac{\frac{z}{\tanh (z)}-1}{z^{2}}\right||k|^{2} \delta^{2} \frac{a_{22}}{a_{11}},
$$

and, for $j \in\{1,2\}$,

$$
0<\frac{\left(\hat{s}_{j}+\hat{f}^{\mathrm{red}}\right)}{\left(\hat{s}_{2}+\hat{g}^{\mathrm{red}}\right)\left(\hat{s}_{1}+\hat{f}^{\mathrm{red}}\right)+\left(\hat{s}_{1}+\hat{g}^{\mathrm{red}}\right)\left(\hat{s}_{2}+\hat{f}^{\mathrm{red}}\right)} \leq \frac{1}{\hat{s}_{\{j+1\}}+\hat{g}^{\mathrm{red}}} \leq \frac{1}{\hat{g}^{\mathrm{red}}} \leq \frac{\delta}{a_{11}},
$$

where we have used the positivity of the occurring coefficients. We see that the coefficient in front of the sum $\left(\hat{\gamma}_{2} \hat{u}_{2}+\hat{\gamma}_{1} \hat{u}_{1}\right)$ is of third order in $\delta$, whereas the coefficient in front of the difference $\left(\hat{u}_{2}-\hat{u}_{1}\right)$ is only of second order. But we can gain one order in $\delta$ by using (46),

$$
\begin{aligned}
\left|\hat{\gamma}_{1} \hat{u}_{1}-\hat{\gamma}_{2} \hat{u}_{2}\right| & =\frac{\left|\hat{s}_{1} \hat{\gamma}_{1} \hat{u}_{1}-\hat{s}_{2} \hat{\gamma}_{2} \hat{u}_{2}+\widehat{\mathcal{R}_{1}\left(h_{1}\right)}-\widehat{\mathcal{R}_{2}\left(h_{2}\right)}\right|}{\left|\hat{g}^{\mathrm{ex}}\right|} \\
& \leq \frac{\delta}{a_{11}}\left(\left(|k|+\frac{1}{L_{1}-\delta}\right)\left|\hat{\gamma}_{1} \hat{u}_{1}\right|+\left(|k|+\frac{1}{L_{2}-\delta}\right)\left|\hat{\gamma}_{2} \hat{u}_{2}\right|+\left|\widehat{\mathcal{R}_{1}\left(h_{1}\right)}-\widehat{\mathcal{R}_{2}\left(h_{2}\right)}\right|\right),
\end{aligned}
$$

by means of inequality (49). Gathering these inequalities, we obtain for $j \in\{1,2\}$,

$$
\left|\hat{\gamma}_{j} \hat{e}_{j}\right| \leq C \delta^{3}|k|^{2}\left((|k|+1)\left(\left|\hat{\gamma}_{1} \hat{u}_{1}\right|+\left|\hat{\gamma}_{2} \hat{u}_{2}\right|\right)+\left|\widehat{\mathcal{R}_{1}\left(h_{1}\right)}-\widehat{\mathcal{R}_{2}\left(h_{2}\right)}\right|\right)
$$

For the errors in physical space,

$$
e_{j}=\frac{1}{\sqrt{2 \pi}} \int_{-\infty}^{\infty} \hat{e}_{j} e^{i k y} \mathrm{~d} k=u_{j}-u_{j}^{\mathrm{red}}
$$

we have

$$
\begin{aligned}
\left\|\gamma_{j} e_{j}\right\|_{H^{\frac{1}{2}(\mathbb{R})}}= & \left(\int_{\mathbb{R}} \sqrt{1+k^{2}}\left|\hat{\gamma}_{j} \hat{e}_{j}\right|^{2} \mathrm{~d} k\right)^{\frac{1}{2}} \\
\leq & C \delta^{3}\left[\left(\int_{\mathbb{R}} \sqrt{1+k^{2}}\left(|k|^{6}+1\right)\left(\left|\hat{\gamma}_{1} \hat{u}_{1}\right|+\left|\hat{\gamma}_{2} \hat{u}_{2}\right|\right)^{2} \mathrm{~d} k\right)^{\frac{1}{2}}\right. \\
& \left.+\left(\int_{\mathbb{R}} \sqrt{1+k^{2}}|k|^{4}\left(\left|\widehat{\mathcal{R}_{1}\left(h_{1}\right)}\right|+\left|\widehat{\mathcal{R}_{2}\left(h_{2}\right)}\right|\right)^{2} \mathrm{~d} k\right)^{\frac{1}{2}}\right] \\
\leq & C \delta^{3}\left[\sum_{j=1}^{2}\left(\int_{\mathbb{R}}\left(1+k^{2}\right)^{\frac{7}{2}}\left|\hat{\gamma}_{j} \hat{u}_{j}\right|^{2} \mathrm{~d} k\right)^{\frac{1}{2}}+\sum_{j=1}^{2}\left(\int_{\mathbb{R}}\left(1+k^{2}\right)^{\frac{5}{2}}\left|\widehat{\mathcal{R}_{j}\left(h_{j}\right)}\right|^{2} \mathrm{~d} k\right)^{\frac{1}{2}}\right] \\
= & C \delta^{3}\left(\left\|\gamma_{1} u_{1}\right\|_{H^{\frac{7}{2}(\mathbb{R})}}+\left\|\gamma_{2} u_{2}\right\|_{H^{\frac{7}{2}(\mathbb{R})}}+\left\|\mathcal{R}_{1}\left(h_{1}\right)\right\|_{H^{\frac{5}{2}(\mathbb{R})}}+\left\|\mathcal{R}_{2}\left(h_{2}\right)\right\|_{H^{\frac{5}{2}(\mathbb{R})}}\right) .
\end{aligned}
$$

Using the (normal) trace and extension inequalities, we then obtain

$$
\left\|e_{j}\right\|_{H^{1}\left(\Omega_{j}\right)} \leq\left\|\gamma_{j} e_{j}\right\|_{H^{\frac{1}{2}(\mathbb{R})}} \leq C \delta^{3}\left(\left\|u_{1}\right\|_{H^{4}\left(\Omega_{1}\right)}+\left\|u_{2}\right\|_{H^{4}\left(\Omega_{2}\right)}+\left\|w_{1}\right\|_{H^{5}\left(\Omega_{1}\right)}+\left\|w_{2}\right\|_{H^{5}\left(\Omega_{2}\right)}\right),
$$


with $w_{j}$ solutions to problem (44), which classically induces

$$
\left\|w_{j}\right\|_{H^{5}\left(\Omega_{j}\right)} \leq\left\|h_{j}\right\|_{H^{3}\left(\Omega_{j}\right)} .
$$

Thus we obtain the error estimates

$$
\left\|e_{j}\right\|_{H^{1}\left(\Omega_{j}\right)} \leq\left\|\gamma_{j} e_{j}\right\|_{H^{\frac{1}{2}(\Gamma)}} \leq C \delta^{3}\left(\left\|u_{1}\right\|_{H^{4}\left(\Omega_{1}\right)}+\left\|u_{2}\right\|_{H^{4}\left(\Omega_{2}\right)}+\left\|h_{1}\right\|_{H^{3}\left(\Omega_{1}\right)}+\left\|h_{2}\right\|_{H^{3}\left(\Omega_{2}\right)}\right) .
$$

We can now also obtain a rigorous error estimate for the coupled problem from [19].

Theorem 7.2 (Matrix error estimate for problem [19]) Let $\mathbf{A}_{f}$ be diagonal, $\mathbf{A}_{1}=\mathbf{A}_{2}=$ $\mathbf{I}, \mathbf{b}_{1}=\mathbf{b}_{2}=\mathbf{b}_{f}=0$ and $\eta_{1}=\eta_{2}=\eta_{f}=0$. Let $\left\{u_{1}, u_{2}, u_{f}\right\}$ be solution to $(1)-(4)$. Let $\xi \in\left[\frac{1}{2}, 1\right]$ and $\left\{u_{1}^{\xi}, u_{2}^{\xi}, U_{f}^{\xi}\right\}$ be solution to (39)-(43). Then there exists a constant $C>0$ independent of $\delta$, such that for $j=1,2$ we have the estimate

$$
\left\|u_{j}-u_{j}^{\xi}\right\|_{H^{1}\left(\Omega_{j}\right)} \leq C \delta^{3}\left(\left\|u_{1}\right\|_{H^{4}\left(\Omega_{1}\right)}+\left\|u_{2}\right\|_{H^{4}\left(\Omega_{2}\right)}+\left\|h_{1}\right\|_{H^{3}\left(\Omega_{1}\right)}+\left\|h_{2}\right\|_{H^{3}\left(\Omega_{2}\right)}\right) .
$$

Proof We first eliminate $U_{f}^{\xi}$ by substituting (43) into (41) and obtain the coupling conditions

$$
\begin{aligned}
& \left(1-\frac{2 \xi-1}{2} \frac{\delta}{a_{11}} \partial_{y y}\right)\left(\gamma_{\mathbf{n}_{1}} \mathbf{q}_{1}^{\xi}+\gamma_{\mathbf{n}_{2}} \mathbf{q}_{2}^{\xi}\right)=\delta a_{22} \partial_{y y}\left(\gamma_{2} u_{2}^{\xi}+\gamma_{1} u_{1}^{\xi}\right), \\
& \delta\left(\gamma_{\mathbf{n}_{1}} \mathbf{q}_{1}^{\xi}-\gamma_{\mathbf{n}_{2}} \mathbf{q}_{2}^{\xi}\right)=a_{11}\left(\gamma_{2} u_{2}^{\xi}-\gamma_{1} u_{1}^{\xi}\right),
\end{aligned}
$$

or, in Fourier space,

$$
\begin{aligned}
& \hat{s}_{1} \hat{\gamma}_{1} \hat{u}_{1}^{\xi}+\hat{s}_{2} \hat{\gamma}_{2} \hat{u}_{2}^{\xi}+\widehat{\mathcal{R}_{1}\left(h_{1}\right)}+\widehat{\mathcal{R}_{2}\left(h_{2}\right)}=-\hat{f}^{\xi}\left(\hat{\gamma}_{1} \hat{u}_{1}^{\xi}+\hat{\gamma}_{2} \hat{u}_{2}^{\xi}\right), \\
& \hat{s}_{1} \hat{\gamma}_{1} \hat{u}_{1}^{\xi}-\hat{s}_{2} \hat{\gamma}_{2} \hat{u}_{2}^{\xi}+\widehat{\mathcal{R}_{1}\left(h_{1}\right)}-\widehat{\mathcal{R}_{2}\left(h_{2}\right)}=\hat{g}^{\xi}\left(\hat{\gamma}_{2} \hat{u}_{2}^{\xi}-\hat{\gamma}_{1} \hat{u}_{1}^{\xi}\right)
\end{aligned}
$$

where

$$
\hat{f}^{\xi}:=\frac{\delta a_{11} a_{22} k^{2}}{a_{11}+\delta^{2} a_{22} k^{2}(2 \xi-1)}, \quad \hat{g}^{\xi}:=\frac{a_{11}}{\delta}
$$

We have $\hat{g}^{\text {red }}-\hat{g}^{\xi}=0$ and

$$
\hat{f}^{\mathrm{red}}-\hat{f}^{\xi}=\frac{\delta^{3} a_{22}^{2} k^{4}(2 \xi-1)}{a_{11}+\delta^{2} a_{22} k^{2}(2 \xi-1)} .
$$

Further

$$
\left|\hat{f}-\hat{f}^{\xi}\right| \leq\left|\hat{f}-\hat{f}^{\mathrm{red}}\right|+\left|\hat{f}^{\mathrm{red}}-\hat{f}^{\xi}\right| \leq|k|^{4} \delta^{3}\left(\sup _{z \in \mathbb{R}}\left|\frac{\tanh z-z}{z^{3}}\right| \frac{a_{22}^{2}}{a_{11}}+(2 \xi-1) \frac{a_{22}^{2}}{a_{11}}\right) .
$$

The rest of the proof is as in the proof of Theorem 7.1.

Similarly as above, we can now derive estimates for the error in the reconstructed fracture solutions of the reduced models.

Theorem 7.3 (Fracture error estimates) Let $\mathbf{A}_{f}$ be diagonal, $\mathbf{A}_{1}=\mathbf{A}_{2}=\mathbf{I}, \mathbf{b}_{1}=\mathbf{b}_{2}=$ $\mathbf{b}_{f}=0$ and $\eta_{1}=\eta_{2}=\eta_{f}=0$. Let $\left\{u_{1}, u_{2}, u_{f}\right\}$ be solution to (1)-(4) and $\left\{u_{1}^{\text {red }}, u_{2}^{\text {red }}\right\}$ be solution to (5), (6), (20), (21). Let $u_{f}^{\text {red,0 }}, U_{f}^{\text {red,0 }}, u_{f}^{\text {red,2 }}, U_{f}^{\text {red,2 }}$ be defined according to Section 5. Then

1. There exist constants $C, c>0$ independent of $\delta$, such that

$$
\begin{aligned}
& \left.\begin{array}{l}
\left\|\left.u_{f}\right|_{x=0}-u_{f}^{\mathrm{red}, 0}\right\|_{H^{\frac{1}{2}}(\mathbb{R})} \\
\left\|U_{f}-U_{f}^{\mathrm{red}, 0}\right\|_{H^{\frac{1}{2}}(\mathbb{R})}
\end{array}\right\} \\
& \leq c \delta^{2}\left(\left\|u_{1}\right\|_{H^{3}\left(\Omega_{1}\right)}+\left\|u_{2}\right\|_{H^{3}\left(\Omega_{2}\right)}\right)+C \delta^{3}\left(\left\|u_{1}\right\|_{H^{4}\left(\Omega_{1}\right)}+\left\|u_{2}\right\|_{H^{4}\left(\Omega_{2}\right)}+\left\|h_{1}\right\|_{H^{3}\left(\Omega_{1}\right)}+\left\|h_{2}\right\|_{H^{3}\left(\Omega_{2}\right)}\right) \text {. }
\end{aligned}
$$


2. There exists a constant $C>0$ independent of $\delta$, such that

$$
\begin{aligned}
& \left.\begin{array}{l}
\left\|\left.u_{f}\right|_{x=0}-u_{f}^{\mathrm{red}, 2}\right\|_{H^{\frac{1}{2}}(\mathbb{R})} \\
\left\|U_{f}-U_{f}^{\mathrm{red}, 2}\right\|_{H^{\frac{1}{2}}(\mathbb{R})}
\end{array}\right\} \\
& \leq C \delta^{3}\left(\left\|u_{1}\right\|_{H^{4}\left(\Omega_{1}\right)}+\left\|u_{2}\right\|_{H^{4}\left(\Omega_{2}\right)}+\left\|h_{1}\right\|_{H^{3}\left(\Omega_{1}\right)}+\left\|h_{2}\right\|_{H^{3}\left(\Omega_{2}\right)}\right) \text {. }
\end{aligned}
$$

Proof Leading order approximation of $u_{f}(0, y)$ : the leading order reconstructed fracture solution is

$$
u_{f}^{\mathrm{red}, 0}=\frac{u_{1}^{\mathrm{red}}+u_{2}^{\mathrm{red}}}{2} .
$$

Following the calculations in Section 5, we obtain for the error of the Fourier coefficients

$$
\begin{aligned}
\left|\hat{u}_{f}(0, k)-\hat{u}_{f}^{\mathrm{red}, 0}(k)\right| & =\left|\frac{\hat{\gamma}_{1} \hat{u}_{1}+\hat{\gamma}_{2} \hat{u}_{2}}{2 \cosh \left(\delta \sqrt{\frac{a_{22}}{a_{11}} k^{2}}\right)}-\frac{\hat{\gamma}_{1} \hat{u}_{1}^{\mathrm{red}}+\hat{\gamma}_{2} \hat{u}_{2}^{\mathrm{red}}}{2}\right| \\
& \leq \frac{1-\cosh \left(\delta \sqrt{\frac{a_{22}}{a_{11}} k^{2}}\right)}{2 \cosh \left(\delta \sqrt{\frac{a_{22}}{a_{11}} k^{2}}\right)}\left|\hat{\gamma}_{1} \hat{u}_{1}+\hat{\gamma}_{2} \hat{u}_{2}\right|+\frac{\left|\hat{\gamma}_{1} \hat{e}_{1}+\hat{\gamma}_{2} \hat{e}_{2}\right|}{2} \\
& \leq \sup _{z \in \mathbb{R}}\left|\frac{1}{\frac{\cosh z}{z^{2}}-1}\right| \delta^{2} \frac{a_{22}}{a_{11}} k^{2}\left|\hat{\gamma}_{1} \hat{u}_{1}+\hat{\gamma}_{2} \hat{u}_{2}\right|+\frac{\left|\hat{\gamma}_{1} \hat{e}_{1}+\hat{\gamma}_{2} \hat{e}_{2}\right|}{2},
\end{aligned}
$$

and therefore,

$$
\begin{aligned}
& \left\|u_{f}(0, \cdot)-u_{f}^{\mathrm{red}, 0}\right\|_{H^{\frac{1}{2}(\Gamma)}}=\left(\int_{\mathbb{R}} \sqrt{1+k^{2}}\left|\hat{u}_{f}(0, \cdot)-\hat{u}_{f}^{\mathrm{red}, 0}\right|^{2} \mathrm{~d} k\right)^{\frac{1}{2}} \\
& \leq c \delta^{2}\left(\int_{\mathbb{R}} \sqrt{1+k^{2}} k^{4}\left|\hat{\gamma}_{1} \hat{u}_{1}+\hat{\gamma}_{2} \hat{u}_{2}\right|^{2} \mathrm{~d} k\right)^{\frac{1}{2}}+C \delta^{3}\left(\int_{\mathbb{R}} \sqrt{1+k^{2}}\left|\hat{\gamma}_{1} \hat{e}_{1}+\hat{\gamma}_{2} \hat{e}_{2}\right|^{2} \mathrm{~d} k\right)^{\frac{1}{2}} \\
& \leq c \delta^{2}\left\|\gamma_{1} u_{1}+\gamma_{2} u_{2}\right\|_{H^{\frac{5}{2}(\Gamma)}}+C\left\|\gamma_{1} e_{1}+\gamma_{2} e_{2}\right\|_{H^{\frac{1}{2}}(\Gamma)} \\
& \leq c \delta^{2}\left(\left\|u_{1}\right\|_{H^{3}\left(\Omega_{1}\right)}+\left\|u_{2}\right\|_{H^{3}\left(\Omega_{2}\right)}\right)+C \delta^{3}\left(\left\|u_{1}\right\|_{H^{4}\left(\Omega_{1}\right)}+\left\|u_{2}\right\|_{H^{4}\left(\Omega_{2}\right)}+\left\|h_{1}\right\|_{H^{3}\left(\Omega_{1}\right)}+\left\|h_{2}\right\|_{H^{3}\left(\Omega_{2}\right)}\right),
\end{aligned}
$$

where we have used an extension inequality as well as inequality (51).

Next-to-next-to-leading order approximation of $u_{f}(0, y)$ : the next-to-next-to-leading order reconstructed fracture solution is

$$
u_{f}^{\mathrm{red}, 2}=\left(\frac{1}{2}+\frac{\delta^{2} a_{22}}{4 a_{11}} \partial_{y y}\right)\left(u_{1}^{\mathrm{red}}+u_{2}^{\mathrm{red}}\right) .
$$

Following the calculations in Section 5, we obtain for the error of the Fourier coefficients

$$
\begin{aligned}
& \left|\hat{u}_{f}(0, k)-\hat{u}_{f}^{\mathrm{red}, 2}(k)\right|=\left|\frac{\hat{\gamma}_{1} \hat{u}_{1}+\hat{\gamma}_{2} \hat{u}_{2}}{2 \cosh \left(\delta \sqrt{\frac{a_{11}}{a_{22}} k^{2}}\right)}-\left(\frac{1}{2}-\frac{\delta^{2} a_{22} k^{2}}{4 a_{11}}\right)\left(\hat{\gamma}_{1} \hat{u}_{1}^{\mathrm{red}}+\hat{\gamma}_{2} \hat{u}_{2}^{\text {red }}\right)\right| \\
& \leq \sup _{z \in \mathbb{R}}\left|\frac{1-\left(1-\frac{z^{2}}{2}\right) \cosh z}{z^{4} \cosh z}\right|\left(\delta \sqrt{\frac{a_{11}}{a_{22}} k^{2}}\right)^{4} \frac{\left|\hat{\gamma}_{1} \hat{u}_{1}+\hat{\gamma}_{2} \hat{u}_{2}\right|}{2}+\left(\frac{1}{2}-\frac{\delta^{2} a_{11}}{4 a_{22}} k^{2}\right)\left(\left|\hat{\gamma}_{1} \hat{e}_{1}+\hat{\gamma}_{2} \hat{e}_{2}\right|\right) .
\end{aligned}
$$

Hence,

$$
\begin{aligned}
& \left\|u_{f}(0, \cdot)-u_{f}^{\mathrm{red}, 2}\right\|_{H^{\frac{1}{2}(\Gamma)}} \\
& \leq \frac{1}{2}\left\|\gamma_{1} e_{1}+\gamma_{2} e_{2}\right\|_{H^{\frac{1}{2}(\Gamma)}}+\frac{\delta^{2} a_{22}}{4 a_{11}}\left\|\gamma_{1} e_{1}+\gamma_{2} e_{2}\right\|_{H^{\frac{5}{2}(\Gamma)}}+c \delta^{4}\left\|\gamma_{1} u_{1}+\gamma_{2} u_{2}\right\|_{H^{\frac{7}{2}}(\Gamma)} \\
& \leq C\left(\delta^{3}+\delta^{5}\right)\left(\left\|u_{1}\right\|_{H^{4}\left(\Omega_{1}\right)}+\left\|u_{2}\right\|_{H^{4}\left(\Omega_{2}\right)}+\left\|h_{1}\right\|_{H^{3}\left(\Omega_{1}\right)}+\left\|h_{2}\right\|_{H^{3}\left(\Omega_{2}\right)}\right) \\
& \quad+c \delta^{4}\left(\left\|u_{1}\right\|_{H^{4}\left(\Omega_{1}\right)}+\left\|u_{2}\right\|_{H^{4}\left(\Omega_{2}\right)}\right)
\end{aligned}
$$


where we have used an extension inequality as well as inequality (51). It is worth to note that, even though the approximation of the fracture solution is formally of order four, the error is of order three, due to the error of the traces at the interfaces of the reduced order matrix solutions.

Leading order approximation of $U_{f}$ : the leading order reconstructed fracture solution is

$$
U_{f}^{\mathrm{red}, 0}=\frac{u_{1}^{\mathrm{red}}+u_{2}^{\mathrm{red}}}{2} .
$$

Let us first remark that from the calculations in Section 5, we obtain

$$
\hat{U}_{f}(k)=\frac{\tanh \left(\delta \sqrt{\frac{a_{22}}{a_{11}} k^{2}}\right)}{2 \delta \sqrt{\frac{a_{22}}{a_{11}} k^{2}}}\left(\hat{\gamma}_{1} \hat{u}_{1}+\hat{\gamma}_{2} \hat{u}_{2}\right)
$$

and therefore,

$$
\begin{aligned}
\left|\hat{U}_{f}(k)-\hat{U}_{f}^{\text {red }, 0}(k)\right| & \leq\left|\frac{\tanh \left(\delta \sqrt{\frac{a_{22}}{a_{11}} k^{2}}\right)-\delta \sqrt{\frac{a_{22}}{a_{11}} k^{2}}}{2 \delta \sqrt{\frac{a_{22}}{a_{11}}}}\left(\hat{\gamma}_{1} \hat{u}_{1}+\hat{\gamma}_{2} \hat{u}_{2}\right)\right|+\frac{\left|\hat{\gamma}_{1} \hat{e}_{1}+\hat{\gamma}_{2} \hat{e}_{2}\right|}{2} \\
& \leq \sup _{z \in \mathbb{R}}\left|\frac{\tanh (z)-z}{z^{3}}\right| \delta^{2} \frac{a_{22}}{a_{11}} k^{2} \frac{\left|\hat{\gamma}_{1} \hat{u}_{1}+\hat{\gamma}_{2} \hat{u}_{2}\right|}{2}+\frac{\left|\hat{\gamma}_{1} \hat{e}_{1}+\hat{\gamma}_{2} \hat{e}_{2}\right|}{2} .
\end{aligned}
$$

The conclusion is now in the same manner as above and will not be repeated.

Next-to-next-to-leading order approximation of $U_{f}$ : the next-to-next-to-leading order reconstructed fracture solution is

$$
U_{f}^{\mathrm{red}, 2}=\left(\frac{1}{2}+\frac{\delta^{2} a_{22}}{6 a_{11}} \partial_{y y}\right)\left(u_{1}^{\mathrm{red}}+u_{2}^{\mathrm{red}}\right) .
$$

Following the calculations in Section 5, we obtain for the error of the Fourier coefficients

$$
\begin{aligned}
\left|\hat{U}_{f}(k)-\hat{U}_{f}^{\mathrm{red}, 2}(k)\right|= & \left|\tanh \left(\delta \sqrt{\frac{a_{22}}{a_{11}} k^{2}}\right)-\left(\frac{1}{2}-\frac{\delta^{2} a_{22} k^{2}}{6 a_{11}}\right)\left(\hat{\gamma}_{1} \hat{u}_{1}^{\mathrm{red}}+\hat{\gamma}_{2} \hat{u}_{2}^{\text {red }}\right)\right| \\
\leq & \sup _{z \in \mathbb{R}}\left|\frac{\tanh (z)-z+\frac{z^{3}}{3}}{z^{5}}\right|\left(\delta \sqrt{\frac{a_{11}}{a_{22}} k^{2}}\right)^{4} \frac{\left|\hat{\gamma}_{1} \hat{u}_{1}+\hat{\gamma}_{2} \hat{u}_{2}\right|}{2} \\
& +\left(\frac{1}{2}-\frac{\delta^{2} a_{11}}{6 a_{22}} k^{2}\right)\left|\hat{\gamma}_{1} \hat{e}_{1}+\hat{\gamma}_{2} \hat{e}_{2}\right| .
\end{aligned}
$$

The conclusion is now in the same manner as above and will not be repeated.

Theorem 7.4 (Fracture error estimates for problem [19]) Let $\mathbf{A}_{f}$ be diagonal, $\mathbf{A}_{1}=$ $\mathbf{A}_{2}=\mathbf{I}, \mathbf{b}_{1}=\mathbf{b}_{2}=\mathbf{b}_{f}=0$ and $\eta_{1}=\eta_{2}=\eta_{f}=0$. Let $\left\{u_{1}, u_{2}, u_{f}\right\}$ be solution to (1)-(4). Let $\xi \in\left[\frac{1}{2}, 1\right]$ and $\left\{u_{1}^{\xi}, u_{2}^{\xi}, U_{f}^{\xi}\right\}$ be solution to (39)-(43). Then

1. For any $\xi \in\left[\frac{1}{2}, 1\right]$, there exist constants $C, c>0$ independent of $\delta$, such that

$$
\begin{aligned}
& \left.\begin{array}{l}
\left\|\left.u_{f}\right|_{x=0}-U_{f}^{\xi}\right\|_{H^{\frac{1}{2}}(\Gamma)} \\
\left\|U_{f}-U_{f}^{\xi}\right\|_{H^{\frac{1}{2}}(\Gamma)}
\end{array}\right\} \\
& \leq c \delta^{2}\left(\left\|u_{1}\right\|_{H^{3}\left(\Omega_{1}\right)}+\left\|u_{2}\right\|_{H^{3}\left(\Omega_{2}\right)}\right)+C \delta^{3}\left(\left\|u_{1}\right\|_{H^{4}\left(\Omega_{1}\right)}+\left\|u_{2}\right\|_{H^{4}\left(\Omega_{2}\right)}+\left\|h_{1}\right\|_{H^{3}\left(\Omega_{1}\right)}+\left\|h_{2}\right\|_{H^{3}\left(\Omega_{2}\right)}\right) \text {. }
\end{aligned}
$$

2. For $\xi=\frac{2}{3}$, there exists a constant $C>0$ independent of $\delta$, such that

$$
\left\|U_{f}-U_{f}^{\xi}\right\|_{H^{\frac{1}{2}}(\Gamma)} \leq C \delta^{3}\left(\left\|u_{1}\right\|_{H^{4}\left(\Omega_{1}\right)}+\left\|u_{2}\right\|_{H^{4}\left(\Omega_{2}\right)}+\left\|h_{1}\right\|_{H^{3}\left(\Omega_{1}\right)}+\left\|h_{2}\right\|_{H^{3}\left(\Omega_{2}\right)}\right) .
$$


3. For $\xi=\frac{3}{4}$, there exists a constant $C>0$ independent of $\delta$, such that

$$
\left\|\left.u_{f}\right|_{x=0}-U_{f}^{\xi}\right\|_{H^{\frac{1}{2}(\Gamma)}} \leq C \delta^{3}\left(\left\|u_{1}\right\|_{H^{4}\left(\Omega_{1}\right)}+\left\|u_{2}\right\|_{H^{4}\left(\Omega_{2}\right)}+\left\|h_{1}\right\|_{H^{3}\left(\Omega_{1}\right)}+\left\|h_{2}\right\|_{H^{3}\left(\Omega_{2}\right)}\right) .
$$

Proof The proof of Theorem 7.3 can be adapted in a straightforward manner to get this result.

Remark 7.1 Theorem 7.4 implies that the model (39)-(43) yields an optimal third order asymptotical approximation $U_{f}^{\xi}$ of

1. $U_{f}=\frac{1}{2 \delta} \int_{-\delta}^{\delta} u_{f}(x, y) \mathrm{d} x$, when chosing $\xi=\frac{2}{3}$, and

2. $u_{f}(0, y)$, when chosing $\xi=\frac{3}{4}$.

\section{Test cases}

We present here a series of test cases in which we study the convergence, for $\delta \rightarrow 0$, of solutions derived by the reduced model to solutions of the equi-dimensional model. We consider the model solved on the full domain, which consists of the Laplace equation $\Delta u_{j}=0$ in the matrix domains $\Omega_{j}, j=1,2$ and a general elliptic model inside the fracture,

$$
-\operatorname{div}\left(\mathbf{A} \nabla u_{f}\right)+\mathbf{b} \cdot \nabla u_{f}+\eta u_{f}, \quad \text { in } \Omega_{f},
$$

together with the coupling conditions

$$
\begin{gathered}
u_{1}(-\delta)=u_{f}(-\delta) \quad \text { and } \quad u_{2}(\delta)=u_{f}(\delta), \\
\partial_{x} u_{1}(-\delta)=\left(a_{11} \partial_{x}+a_{12} \partial_{y}-\frac{b_{1}}{2}\right) u_{f}(-\delta) \quad \text { and } \quad \partial_{x} u_{2}(\delta)=\left(a_{11} \partial_{x}+a_{12} \partial_{y}-\frac{b_{1}}{2}\right) u_{f}(\delta),
\end{gathered}
$$

and compare the solution to those obtained by the reduced models, which consist of the Laplace equation $\Delta u_{j}^{\text {red }}=0$ in $\Omega_{j}, j=1,2$, together with coupling conditions containing the next-toleading-order corrections (CC1, see (20), (21)),

$$
\begin{aligned}
\partial_{x} u_{1}^{\mathrm{red}}(-\delta)-\partial_{x} u_{2}^{\mathrm{red}}(\delta) & =\delta\left(a_{22} \partial_{y y}-b_{2} \partial_{y}-\eta\right)\left(u_{1}^{\mathrm{red}}(-\delta)+u_{2}^{\mathrm{red}}(\delta)\right) \\
+ & \left(a_{21} \partial_{y}-\frac{b_{1}}{2}\right)\left(u_{2}^{\mathrm{red}}(\delta)-u_{1}^{\mathrm{red}}(-\delta)\right), \\
u_{2}^{\mathrm{red}}(\delta)-u_{1}^{\mathrm{red}}(-\delta) & =\delta a_{11}^{-1}\left(\partial_{x} u_{2}^{\mathrm{red}}(\delta)+\partial_{x} u_{1}^{\mathrm{red}}(-\delta)\right)+\delta\left(a_{12} \partial_{y}-\frac{b_{1}}{2}\right)\left(u_{1}^{\mathrm{red}}(-\delta)+u_{2}^{\mathrm{red}}(\delta)\right),
\end{aligned}
$$

which have been shown in Section 7 to have an error of $\mathcal{O}\left(\delta^{3}\right)$ compared to the exact solution, for diffusion problems with diagonal matrix $A$. We use homogeneous Dirichlet boundary conditions at $y= \pm 10$ and non-homogeneous Dirichlet boundary conditions with values $\pm \cos (\pi y / 20)$ at $x= \pm 10$. In order to not have to repeat parameter choices every time, we assume that the default setting of the fracture parameters is

$$
a_{11}=1, a_{22}=1, a_{12}=0, a_{21}=0, b_{1}=0, b_{2}=0, \eta=0,
$$

and we indicate which of the parameters we modified only in each test case. The errors we measure in the matrix and fracture domains are

$$
\begin{gathered}
e r_{m}=\sum_{j=1}^{2}\left\|u_{j}-u_{j}^{\mathrm{red}}\right\|_{L^{\infty}\left(\Omega_{j}\right)}, \\
e r_{f}=\left\|\frac{1}{2 \delta} \int_{-\delta}^{\delta} u_{f} \mathrm{~d} x-\frac{1}{2}\left(\left.u_{1}^{\mathrm{red}}\right|_{x=-\delta}+\left.u_{2}^{\mathrm{red}}\right|_{x=\delta}\right)\right\|_{L^{\infty}(\mathbb{R})} .
\end{gathered}
$$


(a)

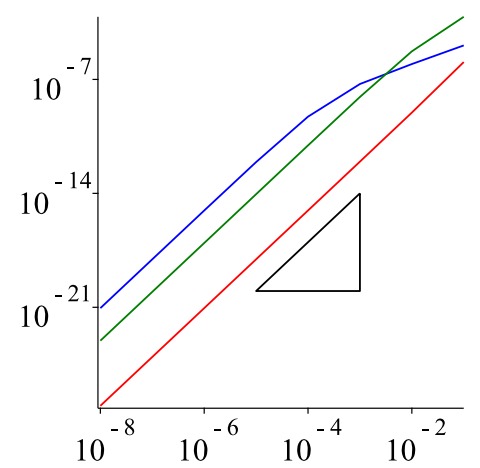

(b)

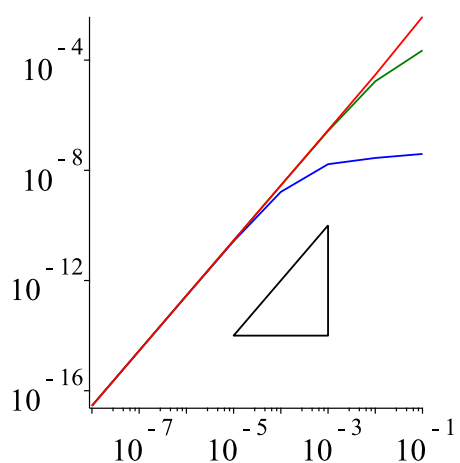

(c)

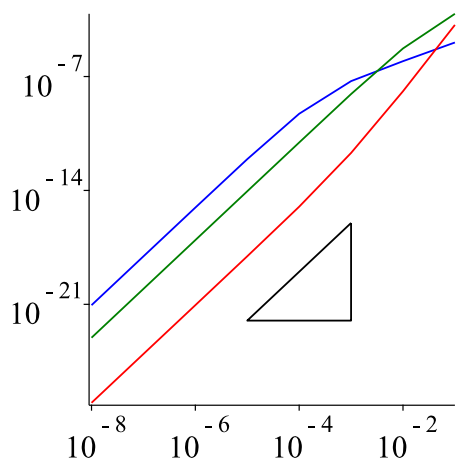

Figure 2: Isotropic Darcy flow with one fracture. The plots show the convergence of the reduced model analytical solutions to the equi-dimensional model w.r.t. the fracture width for fracture permeabilities $a_{11}=a_{22} \in\left\{10^{-3}\right.$ (red curve), 10 (green curve), $10^{3}$ (blue curve) $\}$ and a unit matrix permeability. (a) Error $e r_{m}$ of the matrix solution. (b) Error $e r_{f}$ of the leading order reconstructed fracture solution. (c) Error of the next-to-next-to-leading order reconstructed fracture solution. The reference triangles indicate a cubic slope in (a) and (c) and a quadratic slope in (b).

\subsection{Analytical solutions}

To separate numerical errors from model errors, we start by considering isotropic diffusion in the fracture, i.e. $a_{11}=a_{22} \in \mathbb{R}^{+}$, and we are looking for exact solutions of the equi-dimensional model of the form

$$
u_{j}(x, y)=w_{j}(x) v_{j}(y), \quad j=1,2, f .
$$

For simplicity we choose

$$
v_{1}(y)=v_{2}(y)=v_{f}(y)=\cos \left(\lambda\left(y-a_{y}\right)\right), \quad \lambda=\frac{2 \pi}{\left|b_{y}-a_{y}\right|},
$$

in $\Omega_{1}=\left(a_{x},-\delta\right) \times\left(a_{y}, b_{y}\right), \Gamma=(-\delta, \delta) \times\left(a_{y}, b_{y}\right)$ and $\Omega_{2}=\left(\delta, b_{x}\right) \times\left(a_{y}, b_{y}\right)$, respectively, which satisfy homogeneous Neumann boundary conditions at the $y$-boundary. Then, since $\Delta u_{j}=0$, we have

$$
w_{j}(x)=\alpha_{j} \sinh (\lambda x)+\beta_{j} \cosh (\lambda x), \quad \alpha_{j}, \beta_{j} \in \mathbb{R} .
$$

From the coupling conditions (3), (4), and from the (consistent!) Dirichlet boundary conditions at the $x$-boundary, we obtain the values of the parameters $\alpha_{j}, \beta_{j}$.

For the reduced model, we similarly derive closed form solutions

$$
u_{j}^{\mathrm{red}}(x, y)=w_{j}^{\mathrm{red}}(x) v_{j}^{\mathrm{red}}(y), \quad j=1,2,
$$

in $\Omega_{1}$ and $\Omega_{2}$, with

$$
v_{1}^{\mathrm{red}}(y)=v_{2}^{\mathrm{red}}(y)=v_{f}^{\mathrm{red}}(y)=\cos \left(\lambda\left(y-a_{y}\right)\right), \quad \lambda=\frac{2 \pi}{\left|b_{y}-a_{y}\right|},
$$

and

$$
w_{j}^{\text {red }}(x)=\alpha_{j}^{\text {red }} \sinh (\lambda x)+\beta_{j}^{\text {red }} \cosh (\lambda x), \quad \alpha_{j}^{\text {red }}, \beta_{j}^{\text {red }} \in \mathbb{R} .
$$

From the coupling conditions (20), (21), and from the Dirichlet boundary conditions at the $x$-boundary, we obtain the values of the parameters $\alpha_{j}^{\text {red }}, \beta_{j}^{\text {red }}$.

Figure 2 shows that for low and mid diffusion in the fracture, optimal convergence can be observed for moderate values of $\delta$ already, while for high diffusion in the fracture, this only occurs for very small $\delta$. 


\subsection{Numerical solutions}

We present now numerical tests obtained on Cartesian grids with a classical second order finite difference scheme.

\subsection{1 $\delta$-independent parameters}

We show in Figure 3 the matrix and fracture errors $e r_{m}$ and $e r_{f}$ defined in (55) and (56) for the model coefficients defined in (54), by modifying the values to $a_{11}=0.001$ (top), $a_{22}=1000$ (middle), $b_{2}=200$ (bottom), to obtain anisotropic fracture coefficients. We observe that the orders of convergence for the reduced order matrix and fracture solutions correspond to the orders of convergence predicted by Theorem 7.1 and Theorem 7.3. Consistent with the analytical solutions in Subsection 8.1, for highly diffusive fractures, the solutions enter the regime of predicted convergence only for very small fracture apertures $\delta$. Illustrations of the solutions at $\delta=0.01$ are also given in Figure 3 .

\subsection{2 $\delta$-dependent parameters}

From the reduced order coupling conditions (20), (21), we observe that the fracture aperture, the fracture diffusion coefficients and the fracture tangential advection coefficient never occur isolated, but always in combination either as a fracture resistivity $\frac{a_{11}}{\delta}$ or as fracture conductivities, $\delta a_{22}$ or $\delta b_{2}$. Hence, the asymptotic behavior of the solution is determined by the asymptotic behavior of the generalized fracture coefficients

$$
\frac{a_{11}}{\delta}, \delta a_{22}, \delta b_{2}, a_{12}, a_{21}, b_{1}, \eta
$$

We test our coupling conditions for three different situations: a barrier test case, a conduit test case with diffusion dominant fracture, and a conduit test case with advection dominant fracture. Our results below illustrate well the robustness of our new reduced models.

Barrier test case. In this test case we set $\frac{a_{11}}{\delta}=0.05$, keeping the other parameters as in (54). We show in Figure 4 the matrix and fracture errors $e r_{m}$ and $e r_{f}$, and also the solution in the limit $\delta=0$. From the error plots, we observe an asymptotic linear (matrix) and quadratic (fracture) behavior of the convergence rate, w.r.t. the fracture width.

Conduit test case with diffusion dominant fracture. In this test case we set $\delta a_{22}=10$, keeping the other parameters as in (54). We show in Figure 5 the matrix and fracture errors $e r_{m}$ and $e r_{f}$, and also the solution in the limit $\delta=0$. From the error plots, we observe a linear asymptotic behavior of the convergence rate.

Conduit test case with advection dominant fracture. In this test case we set $\delta b_{2}=2$, keeping again the other parameters as in (54). We show in Figure 6 the matrix and fracture errors $e r_{m}$ and $e r_{f}$, and also the solution in the limit $\delta=0$. We again observe a linear asymptotic behavior of the convergence rate.

\section{Conclusion}

We presented a rigorous derivation of coupling conditions for DFM models for general linear advection-reaction-diffusion problems. The derivation of coupling conditions relies on a Fourier transform of the physical unknowns in direction tangential to the fracture and an elimination of the fracture unknowns in Fourier space by performing a continuous Schur complement. Reduced order coupling conditions are then obtained by straightforward truncation of an expansion in the fracture width. We compared the coupling conditions to a commonly used family of (diffusion) 
a11 $=0.001$



a22 $=1000$

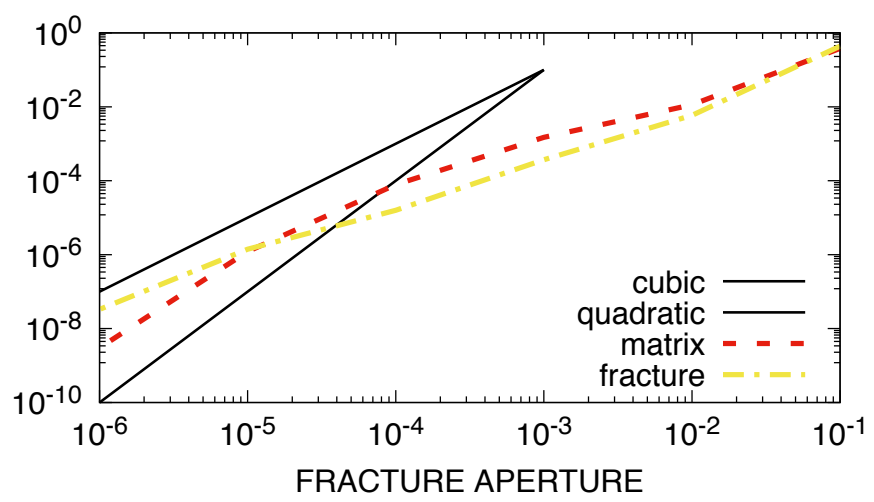

b2 $=200$

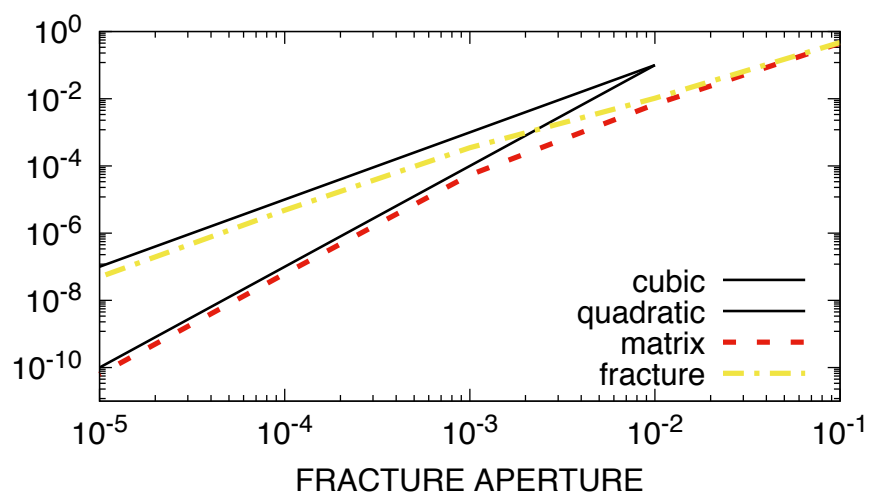

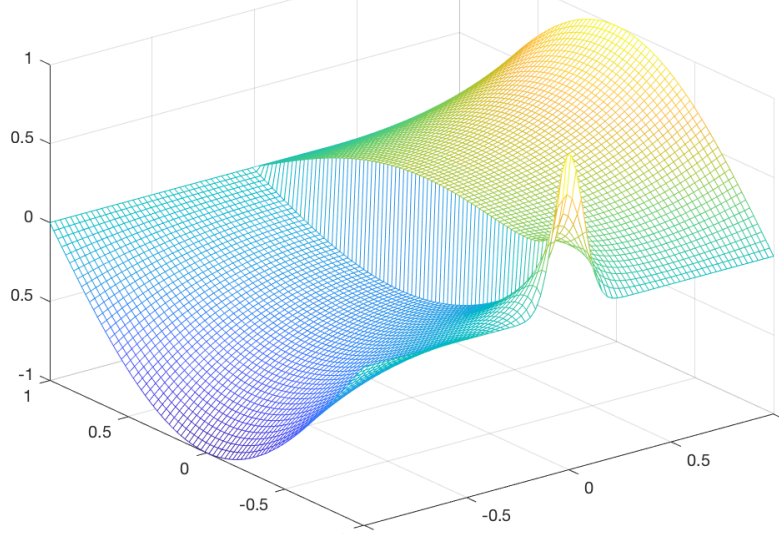
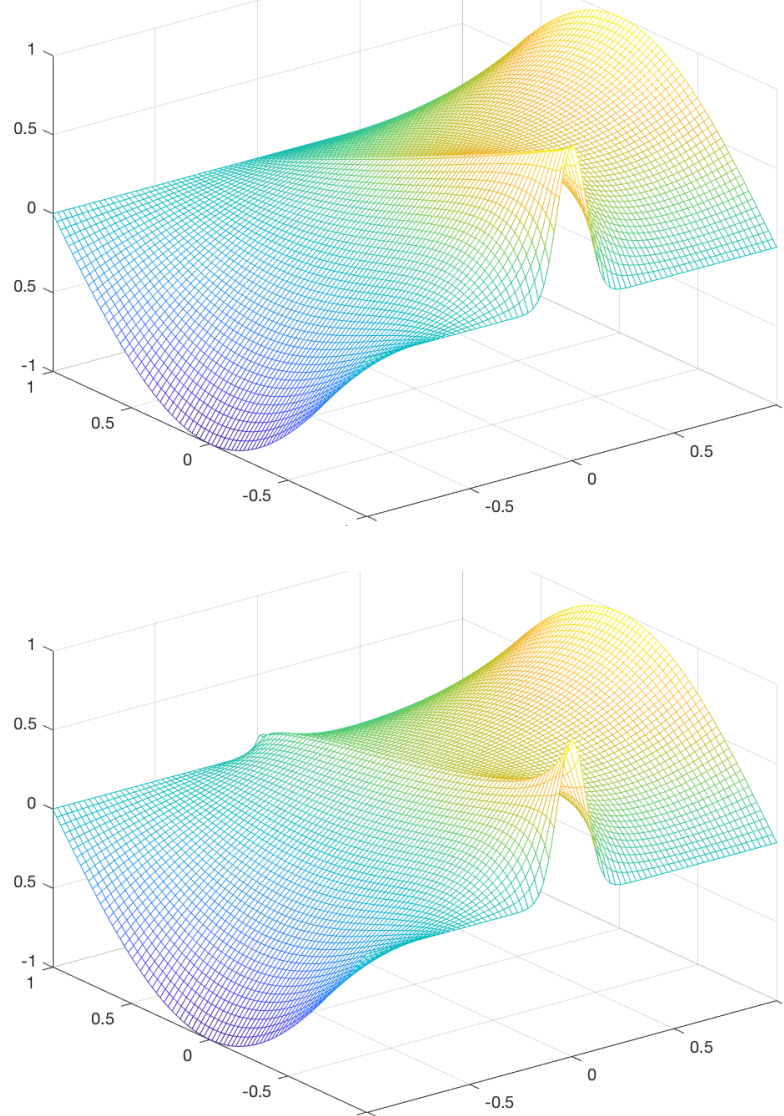

Figure 3: Matrix and fracture errors $e r_{m}$ and $e r_{f}$ for anisotropic fracture coefficients $a_{11}=0.001$, $a_{22}=1000, b_{2}=200$ from top to bottom, the other coefficients being as in (54).

a11/d=0.05

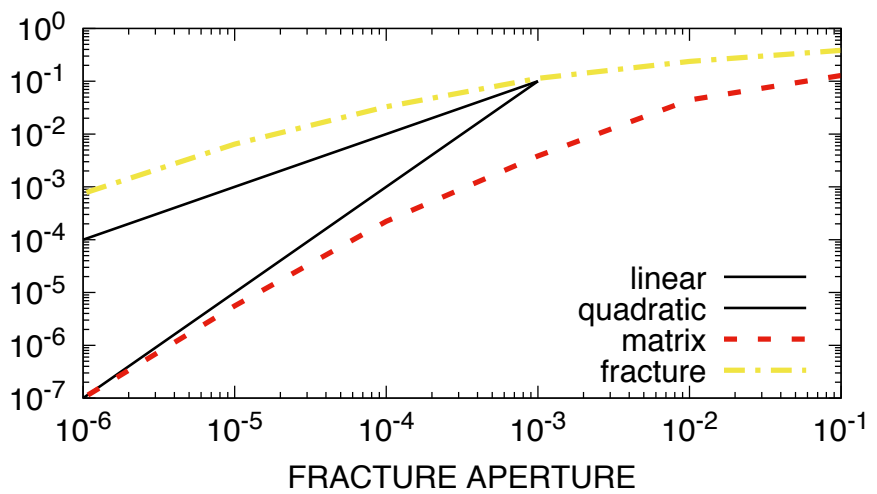

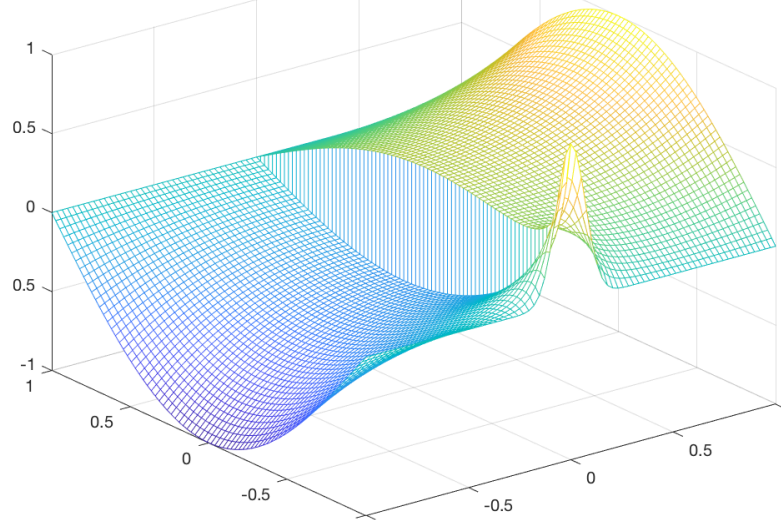

Figure 4: Barrier test. Matrix and fracture error plots $e r_{m}$ and $e r_{f}$ and the solution in the limit $\delta=0$. 

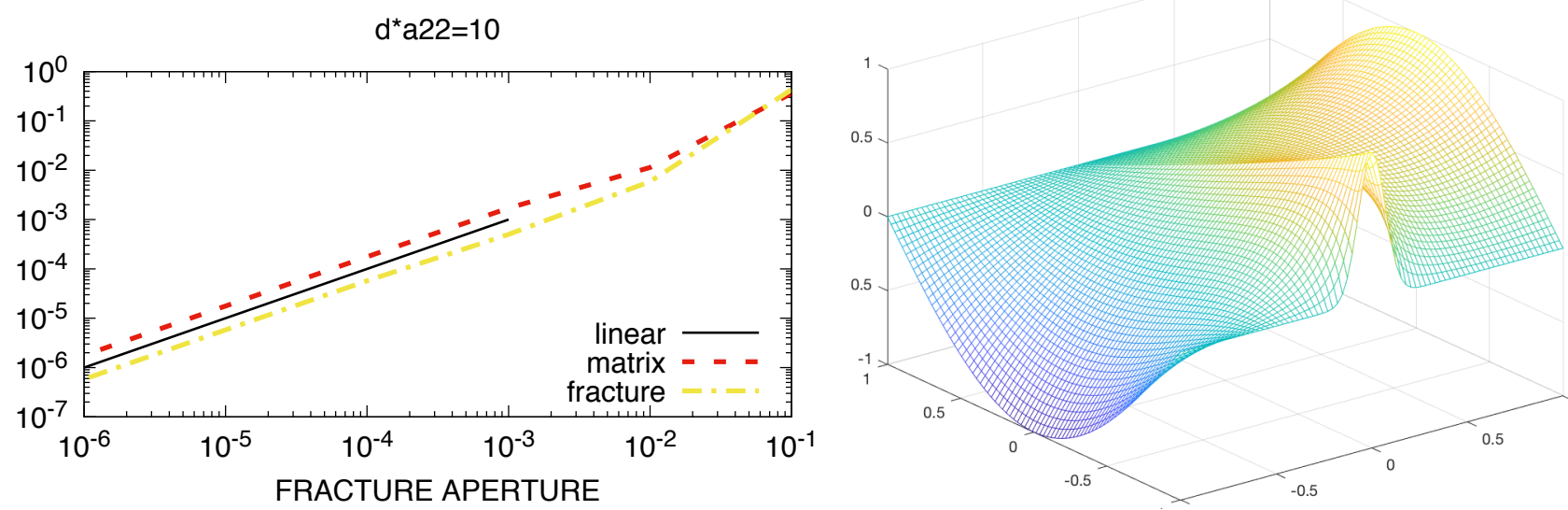

Figure 5: Diffusion dominant conduit test. Matrix and fracture error plots $e r_{m}$ and $e r_{f}$ and the solution in the limit $\delta=0$.
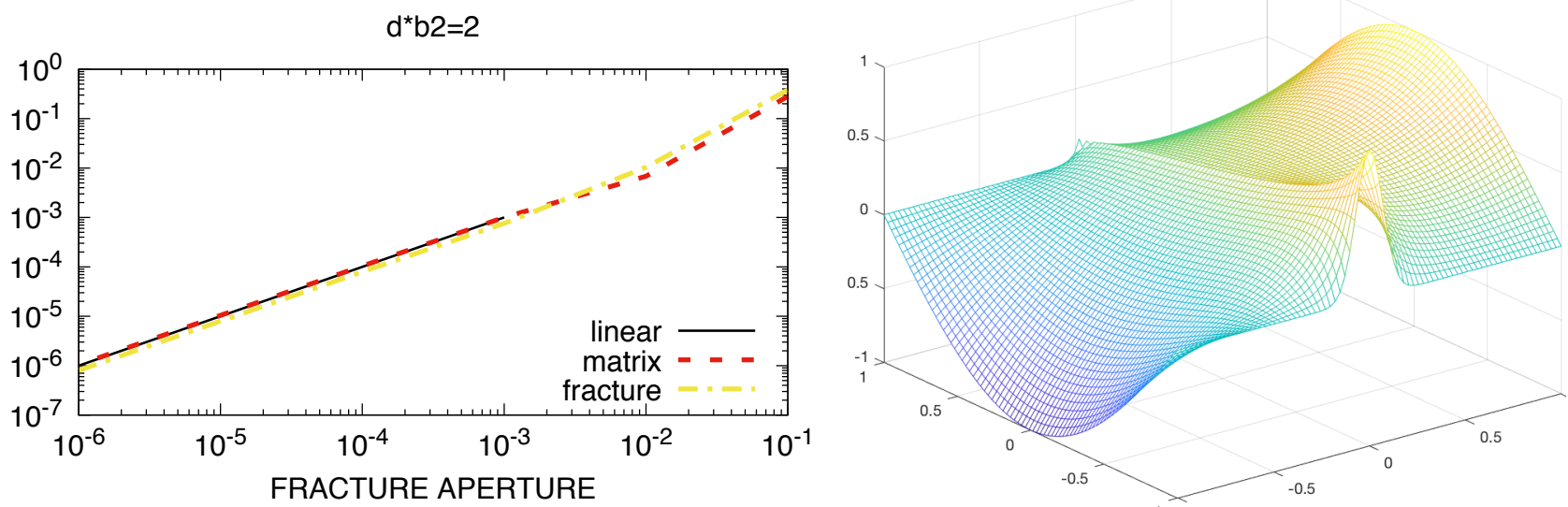

Figure 6: Advection dominant conduit test. Matrix and fracture error plots $e r_{m}$ and $e r_{f}$ and the solution in the limit $\delta=0$. 
models from the literature and obtained correspondence for the coupling conditions truncated after the next-to-leading-order terms. We further derived coupling conditions for the fracture resistivity tending to a constant, to infinity and to zero, and found correspondence to the literature, which contains results for the special case of the Laplace equation only. For the general elliptic models, we showed the well posedness for the reduced models. Furthermore, from the knowledge of the exact solution in Fourier space, we were able to derive error estimates for the reduced model solutions in the norm of fractional Sobolev spaces. Then, we used trace and extension inequalities, in order to obtain error estimates in the $H^{1}$-norm in the matrix domain and in the $H^{\frac{1}{2}}$-norm in the fracture. In particular, we obtained cubic resp. quadratic convergence in $\delta$, for diffusion problems with diagonal matrix $A$. Our rigorous error analysis is currently restricted to these kind of problems. Extensions to more general problems will be presented in future work. Our estimates for the convergence rate of the reduced model solutions has been verified in several numerical tests, and we also presented numerical results which go beyond our analysis, such as for asymptotic solutions in case of a constant fracture conductivity $\delta a_{22}$ or resistivity $\frac{\delta}{a_{11}}$. These results illustrate well the robustness of our new reduced models.

\section{References}

[1] Angot, P., Boyer, F., Hubert, F., 2009 Asymptotic and numerical modelling of flows in fractured porous media, ESAIM Mathematical Modelling and Numerical Analysis 23, 239-275.

[2] Ahmed, E., M., Jaffré, J., Roberts, J. E., A reduced fracture model for two-phase flow with different rock types, Mathematics and Computers in Simulation, 7, pp. 49-70, 2017.

[3] Flemisch, B., Berre, I., Boon, W., Fumagalli, A., Schwenck, N., Scotti, A., Stefansson, I., Tatomir, A., 2018, Benchmarks for single-phase flow in fractured porous media, Adv. Water Resour. 111, 239-258.

[4] Brenner, K., Groza, M., Guichard, C., Lebeau, G. and Masson, R. Gradient discretization of Hybrid-Dimensional Darcy Flows in Fractured Porous Media. Numerische Mathematik, 134,3, pp. 569-609, 2016.

[5] Brenner, K., Groza, M., Guichard, C., Masson, R. Vertex Approximate Gradient Scheme for Hybrid-Dimensional Two-Phase Darcy Flows in Fractured Porous Media. ESAIM Mathematical Modeling and Numerical Analysis, 49, pp. 303-330, 2015.

[6] K. Brenner; J. Hennicker; R. Masson; P. Samier. Gradient discretization of hybriddimensional Darcy flow in fractured porous media with discontinuous pressures at matrixfracture interfaces, IMA Journal of Numerical Analysis, 37,3, pp. 1551-1585, 2016.

[7] K. Brenner, J. Hennicker, R. Masson, P. Samier. Hybrid-dimensional modeling of twophase flow through fractured porous media with enhanced matrix fracture transmission conditions. J. Comp. Physics, 100-124, Vol. 357, 2018.

[8] Brezina, J., Stebel, J., 2016 Analysis of Model Error for a Continuum-Fracture Model of Porous Media Flow, HPCSE 2015. Lecture Notes in Computer Science, vol 9611. Springer, Cham,

[9] Flauraud, E., Nataf, F., Faille, I., Masson, R., 2003, Domain Decomposition for an asymptotic geological fault modeling, Comptes Rendus à l'Académie des Sciences, Mécanique, $331,849-855$.

[10] M. J. Gander, 2006, Optimized Schwarz methods, SIAM J. Numer. Anal., 44, pp. 699-731, 
[11] M. J. Gander, 2008, Schwarz Methods over the Course of Time, ETNA, Vol. 31, pp. 228-255.

[12] M.J. Gander, L. Halpern, C. Japhet and V. Martin, 2009, Viscous Problems with a Vanishing Viscosity Approximation in Subregions: a New Approach Based on Operator Factorization, ESAIM Proc., Vol. 27, pp. 272-288.

[13] M.J. Gander and V. Martin, 2013, An Asymptotic Approach to Compare Coupling Mechanisms for Different Partial Differential Equations, Domain Decomposition Methods in Science and Engineering XX, LNCSE, Springer-Verlag, pp. 359-366.

[14] M.J. Gander, L. Halpern and V. Martin, 2016, A new Algorithm Based on Factorization for Heterogeneous Domain Decomposition, Numerical Algorithms, Vol. 73, No. 1, pp. 167-195.

[15] M.J. Gander, L. Halpern and V. Martin, 2018, Multiscale analysis of heterogeneous domain decomposition methods for time-dependent advection reaction diffusion problems, Journal of Computational and Applied Mathematics, Vol. 344, pp. 904-924.

[16] Julian Hennicker, 2017, Hybrid dimensional modeling of multi-phase Darcy flows in fractured porous media, PhD Thesis, Université Côte d'Azur.

https://tel.archives-ouvertes.fr/tel-01614307

[17] K. Kumar, F. List, I. S. Pop, F. A. Radu, 2019, Formal upscaling and numerical validation of fractured flow models for Richards equation, CMAT Report UP-19-03, Hasselt University.

[18] Matteo Lesinigo, Carlo D'Angelo, Alfio Quarteroni, 2011, A multiscale Darcy-Brinkman model for fluid flow in fractured porous media, Numerische Mathematik, 117 (4). Pages 717-752.

[19] Martin, V., Jaffré, J., Roberts, J. E., 2005, Modeling fractures and barriers as interfaces for flow in porous media, SIAM J. Sci. Comput. 26 (5), 1667-1691.

[20] F. Morales, R. E. Showalter, 2010, The narrow fracture approximation by channeled flow, J. Math. Anal. Appl. 365: 320331.

[21] I. S. Pop, J. Bogers, K. Kumar, 2016, Analysis and upscaling of a reactive transport model in fractured porous media involving nonlinear transmission condition, Vietnam J. Math. 45: 77102 .

[22] E. Sánchez-Palencia, Problèmes de perturbations liés aux phénomènes de conduction à travers des couches minces de grande résistivité, J. Math. Pures et Appl. (9), 53 (1974), pp. 251-269. 